\title{
An exact separation algorithm for unsplittable flow capacitated network design arc-set polyhedron
}

\author{
Liang Chen ${ }^{\star}$. Wei-Kun Chen ${ }^{\dagger}$. \\ Mu-Ming Yang* • Yu-Hong Dai ${ }^{\star}$
}

Received: date / Accepted: date

\begin{abstract}
In this paper, we concentrate on generating cutting planes for the unsplittable capacitated network design problem. We use the unsplittable flow arc-set polyhedron of the considered problem as a substructure and generate cutting planes by solving the separation problem over it. To relieve the computational burden, we show that, in some special cases, a closed form of the separation problem can be derived. For the general case, a brute-force algorithm, called exact separation algorithm, is employed in solving the separation problem of the considered polyhedron such that the constructed inequality guarantees to be facet-defining. Furthermore, a new technique is presented to accelerate the exact separation algorithm, which significantly decreases the number of iterations in the algorithm. Finally, a comprehensive computational study on the unsplittable capacitated network design problem is presented to demonstrate the effectiveness of the proposed algorithm.
\end{abstract}

Keywords Cutting planes · Exact separation - Flow arc-set polyhedron . Unsplittable capacitated network design

Mathematics Subject Classification (2010) 90C11 · 90C27

\section{Introduction}

The unsplittable capacitated network design problem plays an important role in many applications such as telecommunication network design, production

This work was supported by the Chinese Natural Science Foundation (Nos. 11631013, 11331012) and the National 973 Program of China (No. 2015CB856002).

*LSEC, ICMSEC, Academy of Mathematics and Systems Science, Chinese Academy of Sciences, Beijing, China . School of Mathematical Sciences, University of Chinese Academy of Sciences, Beijing, China. Emails: chenliang@lsec.cc.ac.cn, ymm@lsec.cc.ac.cn, dyh@lsec.cc.ac.cn

${ }^{\dagger}$ School of Mathematics and Statistics/Beijing Key Laboratory on MCAACI, Beijing Institute of Technology, Beijing, China Emails: chenweikun@bit.edu.cn (Corresponding author) 
distribution, and express package delivery; see [7,14,19] and the references therein. Given a network, a demand set with its origin-destination pairs of nodes for commodities, and a facility set with different types of facilities (with varying capacities and installation costs), the unsplittable capacitated network design problem is to install integer multiples of facilities on each arc of the network and route the flow of each commodity on a single path such that the total flow cannot exceed the total capacity on each arc, and the sum of facility installation costs and flow routing costs is minimized while meeting the demands of the commodities.

Let $G=(V, E)$ be a directed graph with the node set $V$ and the arc set $E$. Denote $Q$ and $T$ be the sets of commodities and facilities, respectively. The demand of commodity $q \in Q$ from the source node $\zeta^{q}$ to the destination node $\eta^{q}$ is known as $a_{q}$. Suppose that $w_{q}^{i j}$ is the routing cost for commodity $q$ on $\operatorname{arc}(i, j) \in E$. If one module of facility $t \in T$ is installed on $\operatorname{arc}(i, j)$, let $b_{t}$ and $p_{t}^{i j}$ represent the additional capacity and the installation cost on $\operatorname{arc}(i, j)$, respectively. The existing capacity on $\operatorname{arc}(i, j)$ is known as $c^{i j}$. We introduce the binary variable $x_{q}^{i j}$ to denote whether or not commodity $q$ goes through arc $(i, j)$. The variable $y_{t}^{i j}$ denotes the number of facility $t$ installed on arc $(i, j)$. With these notations and variables, the mathematical formulation of the unsplittable capacitated network design problem is

$$
\begin{array}{lll}
\min _{x, y} & \sum_{(i, j) \in E} \sum_{q \in Q} w_{q}^{i j} x_{q}^{i j}+\sum_{(i, j) \in E} \sum_{t \in T} p_{t}^{i j} y_{t}^{i j}, & \\
\text { s.t. } & \sum_{(i, j) \in E} x_{q}^{i j}-\sum_{(j, i) \in E} x_{q}^{j i}=\left\{\begin{aligned}
1, \text { if } i=\zeta^{q} ; \\
-1, \text { if } i=\eta^{q} ; \\
0, \text { otherwise, }
\end{aligned}\right. & \forall q \in Q, i \in V, \\
& \sum_{q \in Q} a_{q} x_{q}^{i j} \leq \sum_{t \in T} b_{t} y_{t}^{i j}+c^{i j}, & \forall(i, j) \in E, \\
& x_{q}^{i j} \in\{0,1\}, y_{t}^{i j} \in \mathbb{Z}_{+}, & \forall q \in Q, t \in T,(i, j) \in E .
\end{array}
$$

In the above formulation, we minimize the sum of facility installation costs and flow routing costs in the objective function (1). Constraint (2) is the flow balance constraint. Constraint (3) is the capacity constraint that requires that the total flow cannot exceed the total capacity on each arc.

Problem (1)-(4) is $\mathcal{N} \mathcal{P}$-hard even for $|Q|=1$ and $|T|=1$ [15]. Hence there is little hope to develop a theoretically efficient algorithm for solving it. Nevertheless, several polyhedral studies of some special cases of the problem have been done in the literature $[2,4,7,8,13,14,19,28,30]$, which suggests us that it is possible to develop a computationally efficient algorithm if the polyhedral structure is well understood. Inspired by this, in this paper, we consider the convex hull of the set related to the capacity constraint on each arc, i.e., the so-called unsplittable flow arc-set polyhedron $P=\operatorname{conv}(X)$ where

$$
X=\left\{(x, y) \in\{0,1\}^{|Q|} \times \mathbb{Z}_{+}^{|T|}: \sum_{q \in Q} a_{q} x_{q} \leq \sum_{t \in T} b_{t} y_{t}+c\right\} .
$$


Here the arc subscripts on variables $x_{t}^{i j}$ and $y_{t}^{i j}$, and parameter $c_{t}^{i j}$ are dropped.

There exist several works studying the unsplittable flow arc-set polyhedron. In particular, Brockmüller et al. [13,14] developed the c-strong inequality for the unsplittable flow arc-set polyhedron when there are only two facilities and the capacity of the second facility is an integer multiple of that of the first one. For problems with a single facility, i.e., $|T|=1$, Atamtürk and Rajan [4] proposed the $k$-split c-strong inequality and the lifted cover inequality. Van Hoesel et al. [30] studied the lower convex envelope inequality. Their computational experiments on the c-strong inequality, the $k$-split c-strong inequality, the lifted cover inequality, and the lower convex envelope inequality demonstrate the effectiveness of integrating these inequalities in a branch-and-cut framework. Benhamiche et al. [8] generalized the c-strong inequality to solve a variant of the unsplittable capacitated network design problem.

Unfortunately, most of these studies are restricted to the unsplittable flow arc-set polyhedron with a single facility or two facilities with divisible capacities. The valid inequalities developed under these assumptions cannot be applied in the context of an arbitrary number of facilities and arbitrary capacities.

In this study, we do not make assumptions on either the number of facilities or the structure of the capacities. Instead, our approach is to develop an exact separation algorithm to solve the separation problem of the unsplittable flow arc-set polyhedron $P$ with an arbitrary number of facilities and arbitrary capacities. More precisely, given a point $(\bar{x}, \bar{y}) \in \mathbb{R}^{|Q|} \times \mathbb{R}^{|T|}$, we want to generate a hyperplane to separate point $(\bar{x}, \bar{y})$ from $P$ or prove that point $(\bar{x}, \bar{y}) \in P$. To do this, we first analyze the coefficients in the nontrivial facet-defining inequality of polyhedron $P$, which is employed in formulating the separation problem as an optimization problem. We prove that the solution of the optimization problem corresponds to a facet-defining inequality of polyhedron $P$. To relieve the computational burden, we show that, in some special cases, a closed form of the optimization problem can be derived. For the general case, the exact separation algorithm, which includes the four steps: preprocessing, row generation, numerical errors, and sequential lifting, is employed in solving the optimization problem. Furthermore, a new technique is presented to accelerate the exact separation algorithm, which significantly decreases the number of iterations in the row generation subroutine. Finally, a comprehensive computational study is presented to test the effectiveness of the proposed algorithm.

It is worth noting that the considered exact separation for the unsplittable flow arc-set polyhedron can be seen as an extension of the exact separation for the 0-1 knapsack polytope; see $[5,6,9,10,11,12,20,31]$ and the references therein. The difference is that the exact separation for the $0-1$ knapsack polytope cannot handle non-binary integer variables, whereas the approach in this paper takes the non-binary integer variables into consideration such that it can be customized to solve the unsplittable capacitated network design problem.

The organization of this paper is as follows. In Sect. 2, we analyze the properties of the nontrivial facet-defining inequalities of polyhedron $P$ and 
formulate the separation problem as an optimization problem. In Sect. 3, We consider some special cases for which a closed form of the optimization problem can be derived. In Sect. 4, we give a framework of the exact separation algorithm including preprocessing in Sect. 4.1, row generation in Sect. 4.2, numerical errors in Sect. 4.3, and sequential lifting in Sect. 4.4. In Sect. 5, we present the numerical results. Finally, in Sect. 6, we give some conclusions and future works.

Throughout this paper, let $e^{i} \in \mathbb{R}^{|Q|}$ and $\boldsymbol{f}^{j} \in \mathbb{R}^{|T|}$ be the $i$-th $|Q|$ dimensional unit vector and $j$-th $|T|$-dimensional unit vector, respectively. Denote $\boldsymbol{e}=(1,1, \ldots, 1)^{\top} \in \mathbb{R}^{|Q|}$ and $\boldsymbol{f}=(1,1, \ldots, 1)^{\top} \in \mathbb{R}^{|T|}$. We use $X_{\mathrm{LP}}$ to denote the linear relaxation of set $X$ obtained by relaxing the integer variables to continuous variables. We assume that $T=\{1, \ldots,|T|\} \neq \varnothing, 0<b_{1} \leq \cdots \leq$ $b_{|T|}$, and $a_{q}>0$ for all $q \in Q$. Without loss of generality, we assume $a^{\top} \boldsymbol{e}-c>0$ since otherwise the capacity constraint in the unsplittable flow arc-set $X$ is redundant.

\section{Separation problem for the unsplittable flow arc-set polyhedron}

In this section, we first study the polyhedral properties of the unsplittable flow arc-set polyhedron $P$. Then we formulate the separation problem over polyhedron $P$ as an optimization problem and prove that there exists an optimal solution which corresponds to a facet-defining inequality of polyhedron $P$.

2.1 Characteristics of the unsplittable flow arc-set polyhedron

We first note that polyhedron $P$ is full dimensional.

Proposition 1 The dimension of polyhedron $P$ is $|Q|+|T|$.

Next, the following characterizations of some vertices and extreme rays of polyhedron $P$ are straightforward.

Proposition $\mathbf{2}$ The extreme rays of polyhedron $P$ are $\left(\mathbf{0}, \boldsymbol{f}^{\mathbf{1}}\right), \ldots,\left(\mathbf{0}, \boldsymbol{f}^{|\boldsymbol{T}|}\right)$.

Proposition 3 The point $\left(x, \rho_{t}(x) \boldsymbol{f}^{\boldsymbol{t}}\right)$ is a vertex of polyhedron $P$ for each $x \in\{0,1\}^{|Q|}$ and $t \in T$, where

$$
\rho_{t}(x)=\max \left\{\left\lceil\left(a^{\top} x-c\right) / b_{t}\right\rceil, 0\right\} .
$$

The initial constraints $x_{q} \geq 0, x_{q} \leq 1, y_{t} \geq 0$, and $a^{\top} x \leq b^{\top} y+c$ are called trivial inequalities of polyhedron $P$. We now present a necessary condition to guarantee the nontrivial inequality

$$
\alpha^{\top} x \leq \beta^{\top} y+\gamma
$$

to be facet-defining for polyhedron $P$. 
Proposition 4 Let (6) be a nontrivial facet-defining inequality of polyhedron $P$. Then

(i) $\alpha_{q} \geq 0$ and $\alpha_{q} \leq\left\lceil a_{q} / b_{t}\right\rceil \beta_{t}$ for each $q \in Q$ and $t \in T$;

(ii) $0<\beta_{t} \leq\left\lceil b_{t} / b_{k}\right\rceil \beta_{k}$ for each $t, k \in T$ with $t \neq k$;

(iii) $\gamma \geq \begin{cases}0, & \text { if } c \geq 0 ; \\ -\left\lceil-c / b_{t}\right\rceil \beta_{t}, \forall t \in T, & \text { otherwise. }\end{cases}$

Proof For notation convenience, denote $F=P \cap\left\{(x, y): \alpha^{\top} x=\beta^{\top} y+\gamma\right\}$.

(i) For each $q \in Q$, since $F$ is a nontrivial facet of polyhedron $P$ and inequality (6) differs from $x_{q} \geq 0$, there exists a point $\left(x^{(1)}, y^{(1)}\right) \in F$ with $x_{q}^{(1)}=1$ and

$$
\alpha^{\top} x^{(1)}=\beta^{\top} y^{(1)}+\gamma .
$$

Since the coefficient $a_{q}>0$, we have $\left(x^{(1)}-e^{q}, y^{(1)}\right) \in P$, and hence point $\left(x^{(1)}-e^{q}, y^{(1)}\right)$ satisfies the valid inequality (6), i.e.,

$$
\alpha^{\top} x^{(1)}-\alpha_{q} \leq \beta^{\top} y^{(1)}+\gamma .
$$

Subtracting (7) from (8), we obtain $\alpha_{q} \geq 0$. On the other hand, as inequality (6) differs from $x_{q} \leq 1$, there exists a point $\left(x^{(2)}, y^{(2)}\right) \in F$ such that $x_{q}^{(2)}=0$. This, combined with the fact that $\left(x^{(2)}+\boldsymbol{e}^{\boldsymbol{q}}, y^{(2)}+\left\lceil a_{q} / b_{t}\right\rceil \boldsymbol{f}^{\boldsymbol{t}}\right) \in P$ for each $t \in T$, indicates that $\alpha_{q} \leq\left\lceil a_{q} / b_{t}\right\rceil \beta_{t}$.

(ii) For each $t \in T$, since inequality (6) differs from $y_{t} \geq 0$, there exists a point $\left(x^{(3)}, y^{(3)}\right) \in F$ such that $y_{t}^{(3)} \geq 1$. Then for all $k \in T \backslash\{t\}$, we have $\left(x^{(3)}, y^{(3)}-\boldsymbol{f}^{t}+\left\lceil b_{t} / b_{k}\right\rceil \boldsymbol{f}^{\boldsymbol{k}}\right) \in P$, which further implies that $\beta_{t} \leq\left\lceil b_{t} / b_{k}\right\rceil \beta_{k}$. For each $t \in T$, as $\left(\mathbf{0}, \boldsymbol{f}^{\boldsymbol{t}}\right)$ is an extreme ray of polyhedron $P$ (see Proposition 2 ), we have $\beta_{t} \geq 0$. If $\beta_{\tau}=0$ for some $\tau \in T$, then $0 \leq \beta_{t} \leq\left\lceil b_{t} / b_{\tau}\right\rceil \beta_{\tau}=0$ for all $t \in T \backslash\{\tau\}$. Hence, inequality (6) reduces to $\alpha^{\top} x \leq \gamma$. This, together with the fact that $\left(\boldsymbol{e},\left\lceil\left(a^{\top} \boldsymbol{e}-c\right) / b_{t}\right\rceil \boldsymbol{f}^{\boldsymbol{t}}\right) \in P$, implies $\alpha^{\top} \boldsymbol{e} \leq \gamma$. However, this means that inequality (6) is dominated by the bound constraints and, thus it cannot define a facet of polyhedron $P$. Therefore, $\beta_{t}>0$ for all $t \in T$.

(iii) If $c \geq 0$, since $(\mathbf{0}, \mathbf{0}) \in P$, then $\gamma \geq 0$; otherwise, as $\left(\mathbf{0},\left\lceil-c / b_{t}\right\rceil \boldsymbol{f}^{\boldsymbol{t}}\right) \in P$, it follows that $\gamma \geq-\left\lceil-c / b_{t}\right\rceil \beta_{t}$ for all $t \in T$.

\subsection{Separation problem}

Given a point $(\bar{x}, \bar{y}) \in \mathbb{R}^{|Q|} \times \mathbb{R}^{|T|}$, the separation problem of polyhedron $P$ is to construct a hyperplane (induced by inequality (6)) separating $(\bar{x}, \bar{y})$ from $P$ strictly, i.e.,

$$
\alpha^{\top} x \leq \beta^{\top} y+\gamma, \forall(x, y) \in P
$$

and

$$
\alpha^{\top} \bar{x}>\beta^{\top} \bar{y}+\gamma
$$

or prove that no such hyperplane exists, i.e., point $(\bar{x}, \bar{y}) \in P$. The separation problem is trivial to be solved if point $(\bar{x}, \bar{y}) \notin X_{\mathrm{LP}}$ since one of the inequalities 
$0 \leq x_{q} \leq 1, q \in Q, y_{t} \geq 0, t \in T$, and $\sum_{q \in Q} a_{q} x_{q} \leq \sum_{t \in T} b_{t} y_{t}+c$ must be violated by this point. Hence, we assume that point $(\bar{x}, \bar{y}) \in X_{\mathrm{LP}}$ throughout this paper. Solving the separation problem is equivalent to solving

$$
\begin{aligned}
v=\max _{\alpha, \beta, \gamma} & \bar{x}^{\top} \alpha-\bar{y}^{\top} \beta-\gamma, \\
\text { s.t. } & x^{\top} \alpha-y^{\top} \beta-\gamma \leq 0, \forall(x, y) \in P, \\
& (\alpha, \beta, \gamma) \in S,
\end{aligned}
$$

where $S$ is a closed convex set which guarantees that problem (9) is bounded. If $v \leq 0$, we prove $(\bar{x}, \bar{y}) \in P$; otherwise, we find the hyperplane $\alpha^{\top} x=\beta^{\top} y+\gamma$ separating $(\bar{x}, \bar{y})$ from $P$ strictly. Let $\left(x^{1}, y^{1}\right), \ldots,\left(x^{u}, y^{u}\right), u \in \mathbb{Z}_{+}$, be the vertices of polyhedron $P$. From the well-known Minkowski-Weyl theorem [25, 32 ] and the description of the extreme rays of polyhedron $P$ in Proposition 2, problem (9) can be reduced to

$$
\begin{aligned}
v=\max _{\alpha, \beta, \gamma} & \bar{x}^{\top} \alpha-\bar{y}^{\top} \beta-\gamma, \\
\text { s.t. } & \left(x^{k}\right)^{\top} \alpha-\left(y^{k}\right)^{\top} \beta-\gamma \leq 0, k=1, \ldots, u, \\
& \beta_{t} \geq 0, \forall t \in T,(\alpha, \beta, \gamma) \in S .
\end{aligned}
$$

Notice that from Proposition 4, we have $\beta_{t}>0$ for all $t \in T$ in the nontrivial facet-defining inequality (6) of polyhedron $P$. Hence, here we consider a special choice of $S$ :

$$
S=\left\{(\alpha, \beta, \gamma): \beta_{1}=1\right\},
$$

where $1 \in T$. Based on this selection, problem (10) further reduces to

$$
\begin{aligned}
\max _{\alpha, \beta, \gamma} & \bar{x}^{\top} \alpha-\bar{y}^{\top} \beta-\gamma, \\
\text { s.t. } & \left(x^{k}\right)^{\top} \alpha-\left(y^{k}\right)^{\top} \beta-\gamma \leq 0, k=1, \ldots, u, \\
& \beta_{1}=1, \beta_{t} \geq 0, \forall t \in T \backslash\{1\} .
\end{aligned}
$$

Below we shall show that problem (12) is feasible and bounded.

Lemma 1 Given a point $(\bar{x}, \bar{y}) \in X_{\mathrm{LP}}$, problem (12) is feasible and bounded.

Proof We only need to show that problem (12) is bounded since the point $(\alpha, \beta, \gamma)=\left(\mathbf{0}, \boldsymbol{f}^{\mathbf{1}}, 0\right)$ is a feasible solution. We shall use the contradiction argument to prove the boundedness of problem (12). Suppose that problem (12) is unbounded. Then there exists a vector $(\Delta \alpha, \Delta \beta, \Delta \gamma) \in \mathbb{R}^{|Q|} \times \mathbb{R}^{|T|} \times \mathbb{R}$ such that $\Delta \beta_{1}=0, \Delta \beta_{t} \geq 0$ for all $t \in T \backslash\{1\}$,

$$
\left(x^{k}\right)^{\top} \Delta \alpha-\left(y^{k}\right)^{\top} \Delta \beta-\Delta \gamma \leq 0, \text { for all } k=1, \ldots, u,
$$

and

$$
\bar{x}^{\top} \Delta \alpha-\bar{y}^{\top} \Delta \beta-\Delta \gamma>0 .
$$

Combining (13) and (14), we have

$$
\left(\bar{x}-x^{k}\right)^{\top} \Delta \alpha>\left(\bar{y}-y^{k}\right)^{\top} \Delta \beta, \text { for all } k=1, \ldots, u .
$$


Define a point $\hat{x} \in \mathbb{R}^{|Q|}$ such that

$$
\hat{x}_{q}= \begin{cases}1, & \text { if } \Delta \alpha_{q} \geq 0 \\ 0, & \text { if } \Delta \alpha_{q}<0\end{cases}
$$

From Proposition 3, point $\left(\hat{x}, \rho_{1}(\hat{x}) \boldsymbol{f}^{\mathbf{1}}\right)$ is a vertex of polyhedron $P$ where $\rho_{1}(\hat{x})$ is defined in (5). By substituting this point into (15) and using $0 \leq \bar{x}_{q} \leq 1$ for all $q \in Q, \bar{y}_{t} \geq 0$ for all $t \in T\left(\right.$ as $\left.(\bar{x}, \bar{y}) \in X_{\mathrm{LP}}\right), \Delta \beta_{1}=0$, and $\Delta \beta_{t} \geq 0$ for all $t \in T \backslash\{1\}$, we have that

$$
\begin{array}{r}
0 \geq \sum_{q \in Q, \Delta \alpha_{q} \geq 0}\left(\bar{x}_{q}-1\right) \Delta \alpha_{q}+\sum_{q \in Q, \Delta \alpha_{q}<0}\left(\bar{x}_{q}-0\right) \Delta \alpha_{q}> \\
\sum_{t \in T \backslash\{1\}}\left(\bar{y}_{t}-0\right) \Delta \beta_{t}+\left(\bar{y}_{1}-\rho_{1}(\hat{x})\right) \Delta \beta_{1} \geq 0,
\end{array}
$$

which leads to a contradiction. Thus problem (12) is bounded.

Lemma 1 guarantees that problem (12) contains at least one optimal solution. Next, we shall show that using the simplex method to solve problem (12), we will obtain a facet-defining inequality of polyhedron $P$.

Theorem 1 Given a point $(\bar{x}, \bar{y}) \in X_{\mathrm{LP}}$, the basic optimal solution $(\alpha, \beta, \gamma)$ of the linear programming problem (12) defines a facet-defining inequality (6) of polyhedron $P$.

Proof Let $(\alpha, \beta, \gamma)$ be a basic optimal solution of problem (12) and (6) be the corresponding inequality. For notation purpose, denote $h=|Q|+|T|$. From the linear programming theory, except the equality $\beta_{1}=1$, there exist another $h$ active constraints at point $(\alpha, \beta, \gamma)$ such that the vectors of the coefficients of the constraints are linearly independent. Without loss of generality, we assume that these constraints are $\beta_{t}=0$ for $t=\ell_{1}, \ldots, \ell_{\tau}\left(\ell_{i} \in T \backslash\{1\}, i=1, \ldots, \tau\right)$, and $\left(x^{k}\right)^{\top} \alpha-\left(y^{k}\right)^{\top} \beta-\gamma=0$ for $k=1, \ldots, h-\tau$. Then

$$
\left[\begin{array}{ccc}
\left(x^{1}\right)^{\top} & \left(y^{1}\right)^{\top} & 1 \\
\vdots & \vdots & \vdots \\
\left(x^{h-\tau}\right)^{\top} & \left(y^{h-\tau}\right)^{\top} & 1 \\
\mathbf{0}^{\top} & \left(\boldsymbol{f}^{\ell_{1}}\right)^{\top} & 0 \\
\vdots & \vdots & \vdots \\
\mathbf{0}^{\top} & \left(\boldsymbol{f}^{\ell_{\tau}}\right)^{\top} & 0
\end{array}\right]\left[\begin{array}{c}
\alpha \\
-\beta \\
-\gamma
\end{array}\right]=\left[\begin{array}{c}
0 \\
\vdots \\
0
\end{array}\right],
$$

where the rank of the coefficient matrix is $h$. By adding the first row to the last $\tau$ rows, we have

$$
\left[\begin{array}{ccr}
\left(x^{1}\right)^{\top} & \left(y^{1}\right)^{\top} & 1 \\
\vdots & \vdots & \vdots \\
\left(x^{h-\tau}\right)^{\top} & \left(y^{h-\tau}\right)^{\top} & 1 \\
\left(x^{1}\right)^{\top} & \left(y^{1}+\boldsymbol{f}^{\ell_{1}}\right)^{\top} & 1 \\
\vdots & \vdots & \vdots \\
\left(x^{1}\right)^{\top} & \left(y^{1}+\boldsymbol{f}^{\ell_{\tau}}\right)^{\top} & 1
\end{array}\right]\left[\begin{array}{c}
\alpha \\
-\beta \\
-\gamma
\end{array}\right]=\left[\begin{array}{c}
0 \\
\vdots \\
0
\end{array}\right] .
$$


It follows immediately that the rank of the new coefficient matrix is also $h$. Hence the points $\left(x^{1}, y^{1}\right),\left(x^{2}, y^{2}\right), \ldots,\left(x^{h-\tau}, y^{h-\tau}\right),\left(x^{1}, y^{1}+f^{\ell_{1}}\right), \ldots,\left(x^{1}, y^{1}+\right.$ $f^{\ell_{\tau}}$ ) are affinely independent. Furthermore, these points satisfy (6) at equality. This, together with the fact that the dimension of polyhedron $P$ is $h$ in Proposition 1, implies that (6) is a facet-defining inequality of polyhedron $P$.

\section{A closed form of the separation problem: sufficient conditions}

Given a point $(\bar{x}, \bar{y}) \in X_{\mathrm{LP}}$, in this section, we consider some special cases for which a closed form of problem (12) can be derived. The analysis result of these special cases will be used as a preprocessing technique to reduce the computational time of solving problem (12) for the general case. We first consider the case with a single facility, i.e., $|T|=1$, and then generalize it to the multifacility case, i.e., $|T| \geq 2$. All proofs of the propositions in this section are given in the appendix.

To simplify the notation, in what follows, we refer problem (12) as the separation problem of polyhedron $P$. We say inequality (6) solves the separation problem (12) for polyhedron $P$ if point $(\alpha, \beta, \gamma)$ is one of its optimal solutions. For notation purpose, denote

$$
r=\max \left\{\left\lceil-c / b_{1}\right\rceil, 0\right\}
$$

and

$$
d \in \operatorname{argmax}_{q}\left\{\bar{x}_{q}: q \in Q\right\} .
$$

\subsection{Single facility}

In this subsection, we consider the single facility case, i.e., $T=\{1\}$. If $Q=\varnothing$, problem (12) is a single variable problem which can be trivially solved, and hence we assume $Q \neq \varnothing$. Throughout this subsection, we restrict to consider the case where (i) the capacity of one module of this facility is larger than or equal to the demand of each commodity; and (ii) if any commodity $q$ goes through this arc, we need to install one more module of this facility on the arc. Mathematically, this can be written as the following two assumptions:

(i) $a_{q} \leq b_{1}$ for all $q \in Q$;

(ii) $b_{1} r+c<a_{q}$ for all $q \in Q$.

We next give a closed form of the separation problem (12) under some conditions in Propositions 5 and 6.

Proposition 5 Let $(\bar{x}, \bar{y}) \in X_{\mathrm{LP}}$. Suppose that $T=\{1\}$, (i), (ii), and

$$
\sum_{q \in Q} a_{q} \leq b_{1}(r+1)+c
$$

hold. The inequality $x_{d} \leq y_{1}-r$ solves the separation problem (12). Moreover, it defines a facet of polyhedron $P$. 
Example 1 Let $X_{1}=\left\{(x, y) \in\{0,1\}^{4} \times \mathbb{Z}_{+}: 11 x_{1}+15 x_{2}+24 x_{3}+50 x_{4} \leq 100 y\right\}$ and $(\bar{x}, \bar{y})=(0.3,0.5,0.9,0.1,0.38)$. Since $r=0, c=0 \leq a_{q}$ for $q=1,2,3,4$, and $\sum_{q=1}^{4} a_{q}=11+15+24+50=100 \leq 100=b_{1}$, by Proposition 5 , inequality $x_{3} \leq y$ is a solution of the separation problem (12) which is violated by point $(\bar{x}, \bar{y})$.

The condition (18) in Proposition 5 requires a large $b$. To see this, suppose $c=0$. Then $r=0$ and the condition reduces to $\sum_{q \in Q} a_{q} \leq b_{1}$, which means one module of the facility is enough to cover all the demands through this arc. Due to this, we derive a closed form under a condition with a smaller $b$ in the following.

Proposition 6 Let $(\bar{x}, \bar{y}) \in X_{\mathrm{LP}}$. Suppose that $T=\{1\}$, (i), (ii), and

$$
\sum_{q \in Q} a_{q}-a_{\bar{q}} \leq b_{1}(r+1)+c<\sum_{q \in Q} a_{q}, \forall \bar{q} \in Q
$$

hold. Then we have the followings.

(a) If $|Q| \leq 2$, the inequality $\sum_{q \in Q} x_{q} \leq y_{1}-r$ solves the separation problem $(12)$.

(b) If $|Q| \geq 3$, one of the following three inequalities solves the separation problem (12), respectively:

$$
\begin{cases}x_{d} \leq y_{1}-r, & \text { if } \frac{\sum_{q \in Q} \bar{x}_{q}}{|Q|-1} \leq \bar{x}_{d} \\ \frac{1}{|Q|-1} \sum_{q \in Q} x_{q} \leq y_{1}-r, & \text { if } \bar{x}_{d}<\frac{\sum_{q \in Q} \bar{x}_{q}}{|Q|-1} \leq 1 \\ \sum_{q \in Q} x_{q} \leq y_{1}-r+|Q|-2, & \text { if } \frac{\sum_{q \in Q} \bar{x}_{q}}{|Q|-1}>1 .\end{cases}
$$

Moreover, in both two cases, the inequalities define facets of polyhedron $P$, respectively.

Example 2 Let $X_{2}=\left\{(x, y) \in\{0,1\}^{4} \times \mathbb{Z}_{+}: 11 x_{1}+15 x_{2}+24 x_{3}+50 x_{4} \leq 90 y\right\}$. It is easy to verify the conditions of Proposition 6 are satisfied. Suppose $(\bar{x}, \bar{y})=(0.4,0.5,0.4,0.4,0.47)$. By simple calculation, it follows that $d=2$, $\bar{x}_{d}=0.5$, and $0.5<\sum_{i=1}^{4} \bar{x}_{i} /(4-1)=\frac{17}{30}<1$. From Proposition 6 , this gives us the inequality

$$
\frac{1}{3}\left(x_{1}+x_{2}+x_{3}+x_{4}\right) \leq y
$$

which cuts off point $(\bar{x}, \bar{y})$. 
3.2 Multifacility

In this subsection, we consider the multifacility case, i.e., $|T| \geq 2$. Throughout this subsection, we restrict to consider the case with (i), (ii), and

(iii) $\sum_{q \in Q} a_{q} \leq b_{t}+c$ for all $t \in T \backslash\{1\}$.

Assumption (iii) means that except the facility $1 \in T$, the capacity of one module of other facilities is large enough to carry out all commodities.

Given a point $(\bar{x}, \bar{y}) \in X_{\mathrm{LP}}$, under assumption (iii), we observe that if $\sum_{t \in T \backslash\{1\}} \bar{y}_{t} \geq 1$, it follows that point $(\bar{x}, \bar{y}) \in P$. Indeed, since point $(\bar{x}, \bar{y}) \in$ $X_{\mathrm{LP}}$, we have $\mathbf{0} \leq \bar{x} \leq \mathbf{1}$. Then, using Proposition 4(i) and the fact that $a_{q}>0$, in order to prove $(\bar{x}, \bar{y}) \in P$, it is enough to show that point $(\boldsymbol{e}, \bar{y}) \in P$. Similarly, by Proposition 4(ii) and $b_{t}>0$ for all $t \in T$, it suffices to show that $(\boldsymbol{e}, \bar{y}) \in P$ with $\sum_{t \in T \backslash\{1\}} \bar{y}_{t}=1$ and $\bar{y}_{1}=0$. The latter is true since $(\boldsymbol{e}, \bar{y})=\sum_{t \in T \backslash\{1\}} \bar{y}_{t}\left(\boldsymbol{e}, \boldsymbol{f}^{\boldsymbol{t}}\right)$, and by assumption (iii), $\left(\boldsymbol{e}, \boldsymbol{f}^{\boldsymbol{t}}\right) \in P$ for each $t \in T \backslash\{1\}$. Therefore, in the remaining of this subsection, we only consider the case $\sum_{t \in T \backslash\{1\}} \bar{y}_{t}<1$.

Let

$$
\tilde{Q}:=\left\{q \in Q: \bar{x}_{q}>\sum_{t \in T \backslash\{1\}} \bar{y}_{t}\right\} .
$$

Based on Propositions 5 and 6, we can derive similar results under the additional assumption (iii) in the case $|T| \geq 2$. This is summarized in Propositions 7 and 8 .

Proposition 7 Let $(\bar{x}, \bar{y}) \in X_{\mathrm{LP}}$. Suppose that $|T| \geq 2$, (i), (ii), (iii), (18) and $\sum_{t \in T \backslash\{1\}} \bar{y}_{t}<1$ hold. Then we have the followings.

(a) If $\tilde{Q}=\varnothing$, the inequality $0 \leq y_{1}+r \sum_{t \in T \backslash\{1\}} y_{t}-r$ solves the separation problem $(12)$.

(b) If $\tilde{Q} \neq \varnothing$, the inequality $x_{d} \leq y_{1}+(r+1) \sum_{t \in T \backslash\{1\}} y_{t}-r$ solves the separation problem (12).

Moreover, in both two cases, the inequalities define facets of polyhedron $P$, respectively.

Proposition 8 Let $(\bar{x}, \bar{y}) \in X_{\mathrm{LP}}$. Suppose that $|T| \geq 2$, (i), (ii), (iii), (19), and $\sum_{t \in T \backslash\{1\}} \bar{y}_{t}<1$ hold. Then we have the followings.

(a) If $\tilde{Q}=\varnothing$, the inequality $0 \leq y_{1}+r \sum_{t \in T \backslash\{1\}} y_{t}-r$ solves the separation problem (12).

(b) If $\tilde{Q} \neq \varnothing$ and $\tilde{Q} \neq Q$, the inequality $x_{d} \leq y_{1}+(r+1) \sum_{t \in T \backslash\{1\}} y_{t}-r$ solves the separation problem (12).

(c) If $\tilde{Q}=Q$ with $|Q| \leq 2$, the inequality $\sum_{q \in Q} x_{q} \leq y_{1}+(r+|Q|) \sum_{t \in T \backslash\{1\}} y_{t}-$ $r$ solves the separation problem (12). 
(d) If $\tilde{Q}=Q$ with $|Q| \geq 3$, one of the following three inequalities solve the separation problem (12), respectively:

$\left\{\begin{array}{lr}x_{d} \leq y_{1}+(r+1) \sum_{t \in T \backslash\{1\}} y_{t}-r, & \text { if } \frac{\sum_{q \in Q} \bar{x}_{q}-\sum_{t \in T \backslash\{1\}} \bar{y}_{t}}{|Q|-1} \leq \bar{x}_{d} ; \\ \frac{1}{|Q|-1} \sum_{q \in Q} x_{q} \leq y_{1}+\left(r+\frac{|Q|}{|Q|-1}\right) \sum_{t \in T \backslash\{1\}} y_{t}-r, & \text { if } \bar{x}_{d}<\frac{\sum_{q \in Q} \bar{x}_{q}-\sum_{t \in T \backslash\{1\}} \bar{y}_{t}}{|Q|-1} \leq 1 ; \\ \sum_{q \in Q} x_{q} \leq y_{1}+(r+2) \sum_{t \in T \backslash\{1\}} y_{t}-r+|Q|-2, & \text { if } \frac{\sum_{q \in Q} \bar{x}_{q}-\sum_{t \in T \backslash\{1\}} \bar{y}_{t}}{|Q|-1}>1 .\end{array}\right.$

Moreover, in all four cases, the inequalities define facets of polyhedron $P$, respectively.

Remark 1 Together with the trivial inequalities, the inequalities listed in Propositions 5-8 describe polyhedron $P$, respectively. Otherwise, suppose that there exists a facet-defining inequality (6) with $\beta_{1}=1$ differing from any of the inequalities in the list of Proposition 5 (Proposition 6, 7, or 8). Then there exists a point $(\bar{x}, \bar{y}) \in P$ fulfilling inequality (6) at equality and the inequalities listed in the proposition are all inactive at this point. Considering the separation problem (12) with point $(\bar{x}, \bar{y})$, we know that the inequalities listed in the proposition cannot solve this problem, which leads to a contradiction.

\section{An exact separation algorithm}

Unlike Sect. 3, in this section, we focus on solving problem (12) without any assumptions on the data. To begin with, we note that since the number of constraints in problem (12) may be exponential, from a computational perspective, it is impractical to solve problem (12) when all the constraints are expressed explicitly. For this reason, we follow [31] to solve problem (12) by decomposing into the following four steps.

1. Preprocessing. In order to save computational time, we implement some preprocessing methods before solving problem (12). Firstly, instead of solving problem (12) directly, we solve a lower dimensional problem to find a violated inequality for the lower dimensional polyhedron. Besides, we avoid solving problem (12) if a most violated inequality is known or point $(\bar{x}, \bar{y}) \in P$ (e.g., by using Propositions 5-8).

2. Row generation. We find a violated inequality or report that no violated one exists by solving problem (12) over the lower dimensional polyhedron with a row generation subroutine.

3. Numerical errors. To avoid numerical instabilities, the constructed inequality is scaled to obtain integral coefficients and the right hand side is recomputed to guarantee its validity.

4. Sequential lifting. The variables that are fixed in the preprocessing step are sequentially lifted to obtain a strong valid inequality for polyhedron $P$. 
4.1 Preprocessing

Given a point $(\bar{x}, \bar{y}) \in X_{\mathrm{LP}}$, let us fix the variables which take values on their bounds and consider the lower dimensional polyhedron $P(\bar{x}, \bar{y})=\operatorname{conv}(X(\bar{x}, \bar{y}))$ where

$$
X(\bar{x}, \bar{y})=\left\{(x, y) \in\{0,1\}^{|\bar{Q}|} \times \mathbb{Z}_{+}^{|\bar{T}|}: \sum_{q \in \bar{Q}} a_{q} x_{q} \leq \sum_{t \in \bar{T}} b_{t} y_{t}+\bar{c}\right\},
$$

$\bar{Q}=\left\{q \in Q: 0<\bar{x}_{q}<1\right\}, \bar{T}=\left\{t \in T: \bar{y}_{t}>0\right\}$, and $\bar{c}=c-\sum_{q \in Q, \bar{x}_{q}=1} a_{q}$. Similar to problem (12), we may solve the separation problem over polyhedron $P(\bar{x}, \bar{y})$ by considering

$$
\begin{aligned}
\max _{\alpha, \beta, \gamma} & \sum_{q \in \bar{Q}} \bar{x}_{q} \alpha_{q}-\sum_{t \in \bar{T}} \bar{y}_{t} \beta_{t}-\gamma, \\
\text { s.t. } & \sum_{q \in \bar{Q}} x_{q}^{k} \alpha_{q}-\sum_{t \in \bar{T}} y_{t}^{k} \beta_{t}-\gamma \leq 0, k=1, \ldots, \bar{u}, \\
& \beta_{1}=1, \beta_{t} \geq 0, \forall t \in \bar{T} \backslash\{1\},
\end{aligned}
$$

where $\left(x^{1}, y^{1}\right), \ldots,\left(x^{\bar{u}}, y^{\bar{u}}\right), \bar{u} \in \mathbb{Z}_{+}$, are the vertices of polyhedron $P(\bar{x}, \bar{y})$. Notice that here we, without loss of generality, assume that $\bar{y}_{1}>0$. Both the numbers of variables and constraints in problem (21) are less than or equal to those in problem (12). Therefore, it can be expected that problem (21) is easier to be solved than problem (12), especially when the number of fixed variables is large.

We next present a preprocessing procedure based on the following observations.

(i) If $\bar{Q}=\varnothing$ and $\bar{T}=\varnothing$, then point $(\bar{x}, \bar{y}) \in P(\bar{x}, \bar{y})$ and, hence there does not exist a violated inequality.

(ii) If $\bar{Q}=\varnothing$ and $\bar{T}=\{1\}$, there is only a single facet-defining inequality $y_{1} \geq\left\lceil-\bar{c} / b_{1}\right\rceil$ of polyhedron $P(\bar{x}, \bar{y})$. If it is violated by point $(\bar{x}, \bar{y})$, we directly move to the sequential lifting step in Sect. 4.4. Otherwise, we have $(\bar{x}, \bar{y}) \in P(\bar{x}, \bar{y})$.

(iii) If

$$
\sum_{q \in \bar{Q}} a_{q}\left\lceil\bar{x}_{q}\right\rceil \leq \sum_{t \in \bar{T}} b_{t}\left\lfloor\bar{y}_{t}\right\rfloor+\bar{c}
$$

then we can conclude that $(\bar{x}, \bar{y}) \in P(\bar{x}, \bar{y})$. To see this, let

$$
K=\left\{(\bar{x}, \bar{y}): x_{q} \in\left\{\left\lfloor\bar{x}_{q}\right\rfloor,\left\lceil\bar{x}_{q}\right\rceil\right\}, \forall q \in \bar{Q}, y_{t} \in\left\{\left\lfloor\bar{y}_{t}\right\rfloor,\left\lceil\bar{y}_{t}\right\rceil\right\}, \forall t \in \bar{T}\right\} .
$$

It follows from $(22)$ that $K \subseteq X(\bar{x}, \bar{y})$. Therefore, we have $(\bar{x}, \bar{y}) \in \operatorname{conv}(K) \subseteq$ $P(\bar{x}, \bar{y})$.

(iv) For polyhedron $P(\bar{x}, \bar{y})$, if one of the conditions in Propositions 5 - 8 is satisfied, we move to the sequential lifting step in Sect. 4.4 or conclude that point $(\bar{x}, \bar{y}) \in P(\bar{x}, \bar{y})$ depending on whether or not one of the inequalities listed in the corresponding proposition is violated by point $(\bar{x}, \bar{y})$. 


\subsection{Row generation}

We now describe the row generation subroutine for solving problem (21). Instead of solving the whole problem (21) with the potentially exponential many constraints, the row generation subroutine solves a problem with a subset of constraints, i.e.,

$$
\begin{aligned}
v(U)=\max _{\alpha, \beta, \gamma} & \sum_{q \in \bar{Q}} \bar{x}_{q} \alpha_{q}-\sum_{t \in \bar{T}} \bar{y}_{t} \beta_{t}-\gamma, \\
\text { s.t. } & \sum_{q \in \bar{Q}} x_{q} \alpha_{q}-\sum_{t \in \bar{T}} y_{t} \beta_{t}-\gamma \leq 0, \forall(x, y) \in U, \\
& \beta_{t} \geq 0, \forall t \in \bar{T} \backslash\{1\}, \beta_{1}=1,
\end{aligned}
$$

in each iteration, where $U \subseteq P(\bar{x}, \bar{y})$. We call (23) the partial separation problem. Apparently, the partial separation problem (23) is a relaxation of problem (21). Therefore, if $v(U) \leq 0$, we conclude that point $(\bar{x}, \bar{y}) \in P(\bar{x}, \bar{y})$; otherwise, the solution $(\bar{\alpha}, \bar{\beta}, \bar{\gamma})$ of problem $(23)$ corresponds to the inequality

$$
\sum_{q \in \bar{Q}} \bar{\alpha}_{q} x_{q} \leq \sum_{t \in \bar{T}} \bar{\beta}_{t} y_{t}+\bar{\gamma}
$$

which is violated by point $(\bar{x}, \bar{y})$. To further test whether or not inequality (24) is valid for $X(\bar{x}, \bar{y})$, we solve the following unbounded integer knapsack problem:

$$
z=\max _{x, y}\left\{\sum_{q \in \bar{Q}} \bar{\alpha}_{q} x_{q}-\sum_{t \in \bar{T}} \bar{\beta}_{t} y_{t}-\bar{\gamma}:(x, y) \in X(\bar{x}, \bar{y})\right\} .
$$

If $z \leq 0$, inequality (24) is valid for $X(\bar{x}, \bar{y})$; otherwise the optimal solution of (25) violates inequality (24), and hence, we add this solution into set $U$ and the procedure continues.

In contrast to the separation problem (12), for which it is shown to be bounded in Lemma 1, the partial separation problem (23) can be unbounded.

Example 3 Let $X^{0}=\left\{(x, y) \in\{0,1\} \times \mathbb{Z}_{+}: 3 x \leq 5 y\right\}$ and $(\bar{x}, \bar{y})=(0.5,0.3)$. Initializing $U=\varnothing$, the partial separation problem (23) reduces to $v(\varnothing)=$ $\max _{\alpha, \gamma}\{0.5 \alpha-0.3-\gamma\}$, which is unbounded.

In order to avoid the partial separation problem (23) to be unbounded, we shall provide some bounds on the variables. As it has been shown in Theorem 1 , the basic optimal solution of problem (21) corresponds to a facet-defining inequality of polyhedron $P(\bar{x}, \bar{y})$. Combining it with Proposition 4 and the fact that $\beta_{1}=1$, we can add the bound constraints

$$
\begin{array}{ll}
0 \leq \alpha_{q} \leq\left\lceil a_{q} / b_{1}\right\rceil, & \forall q \in \bar{Q}, \\
1 \leq \beta_{t} \leq\left\lceil b_{t} / b_{1}\right\rceil, & \forall t \in \bar{T}, \\
\min \left\{-\left\lceil-\bar{c} / b_{1}\right\rceil, 0\right\} \leq \gamma, &
\end{array}
$$


to problem (21) and then derive a stronger partial separation problem:

$$
\begin{array}{rlrl}
v(U)=\max _{\alpha, \beta, \gamma} & \sum_{q \in \bar{Q}} \bar{x}_{q} \alpha_{q}-\sum_{t \in \bar{T}} \bar{y}_{t} \beta_{t}-\gamma, & & \\
\text { s.t. } & \sum_{q \in \bar{Q}} x_{q} \alpha_{q}-\sum_{t \in \bar{T}} y_{t} \beta_{t}-\gamma \leq 0, & & \forall(x, y) \in U, \\
& 0 \leq \alpha_{q} \leq\left\lceil a_{q} / b_{1}\right\rceil, & & \forall q \in \bar{Q}, \\
& 1 \leq \beta_{t} \leq\left\lceil b_{t} / b_{1}\right\rceil, & & \forall t \in \bar{T}, \\
& \gamma \geq \min \left\{-\left\lceil-\bar{c} / b_{1}\right\rceil, 0\right\} . &
\end{array}
$$

Clearly, problem (27) is bounded.

Another issue needed to be addressed is that the optimal solution of the unbounded knapsack problem (25) may not be unique. The inequality derived by some optimal solution may be stronger than those derived by other optimal solutions.

Example \& Let $X_{3}=\left\{(x, y) \in\{0,1\}^{4} \times \mathbb{Z}_{+}: 11 x_{1}+15 x_{2}+24 x_{3}+50 x_{4} \leq 60 y\right\}$. Consider the point $(\bar{x}, \bar{y})=(0.9,0.5,0.7,0.1,0.7)$. After solving the partial separation problem $(27)$ with $U=\{(1,1,1,1,2)\}$, we obtain the solution $(\alpha, \beta, \gamma)=(1,0,1,0,1,0)$ corresponding to the inequality $x_{1}+x_{3} \leq y$. Considering the associated unbounded integer knapsack problem (25), we have two optimal solutions $(1,0,1,0,1)$ and $(1,1,1,0,1)$ which correspond to the inequalities

$$
\alpha_{1}+\alpha_{3}-1-\gamma \leq 0
$$

and

$$
\alpha_{1}+\alpha_{2}+\alpha_{3}-1-\gamma \leq 0,
$$

respectively. Obviously, inequality (29) is stronger than inequality (28). If we add inequality (29) to problem (27), in the next iteration, we will obtain the optimal solution $(\alpha, \beta, \gamma)=(1,0,0,1,1,0)$ corresponding to the inequality $x_{1}+x_{4} \leq y$, which is violated by point $(\bar{x}, \bar{y})$. Moreover, by solving unbounded integer knapsack problem (25), we know that $x_{1}+x_{4} \leq y$ is valid for $P(\bar{x}, \bar{y})$. However, if we add inequality (28) to problem (27), it can be checked that it needs more iterations to solve the separation problem (21).

We see from Example 4 that, to speed up the row generation procedure, among (possible) multiple optimal solutions of the unbounded integer knapsack problem, it is crucial to select one which corresponds to a stronger inequality for problem (27). Due to this, we next describe an iterative approach to modify an existing optimal solution for problem (25) such that the new optimal solution corresponds to a (possibly) stronger constraint in problem (27).

Let $(\tilde{x}, \tilde{y})$ be an optimal solution of the unbounded integer knapsack problem (25). The corresponding inequality in problem (27) is

$$
\sum_{q \in \bar{Q}} \tilde{x}_{q} \alpha_{q}-\sum_{t \in \bar{T}} \tilde{y}_{t} \beta_{t}-\gamma \leq 0 .
$$


Now suppose that $\tilde{x}_{q^{\prime}}=0$ for some $q^{\prime} \in \bar{Q}$ and point $\left(\tilde{x}+e^{q^{\prime}}, \tilde{y}\right) \in X(\bar{x}, \bar{y})$. Notice that as point $(\bar{\alpha}, \bar{\beta}, \bar{\gamma})$ is feasible solution of problem $(27)$, we have $\bar{\alpha}_{q^{\prime}} \geq$ 0 . Hence $\left(\tilde{x}+e^{q^{\prime}}, \tilde{y}\right)$ is also an optimal solution of problem (25) corresponding to the inequality

$$
\sum_{q \in \bar{Q}} \tilde{x}_{q} \alpha_{q}+\alpha_{q^{\prime}}-\sum_{t \in \bar{T}} \tilde{y}_{t} \beta_{t}-\gamma \leq 0,
$$

which is obviously stronger than inequality (30). Furthermore, we can recursively use this argument to strengthen an inequality based on the new optimal solution. We describe this iterative approach in Algorithm 1.

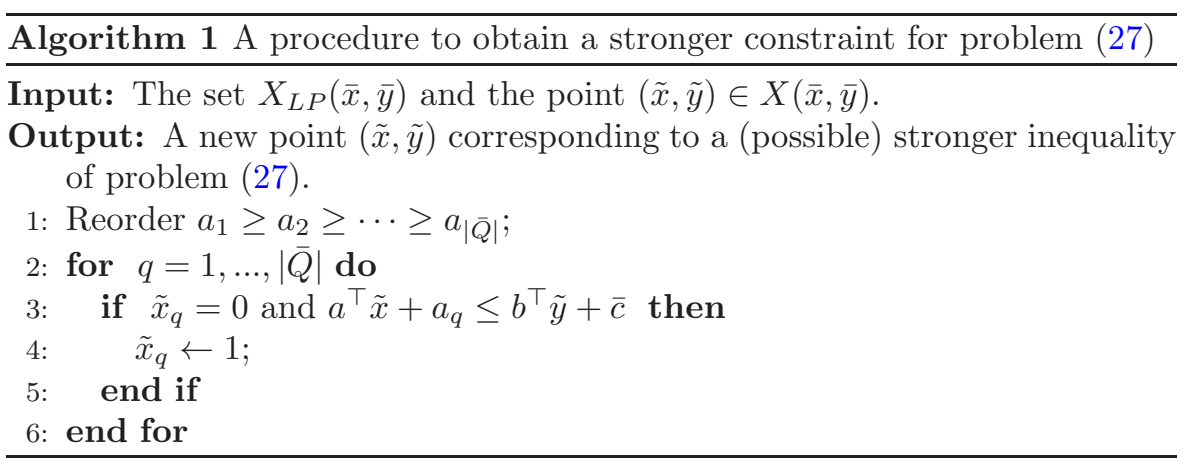

In Algorithm 1, we first sort the variables such that $a_{1} \geq a_{2} \geq \cdots \geq a_{|\bar{Q}|}$ in Step 1 . We then recursively modify a point to a new point which corresponds to a (possibly) stronger constraint for problem (27) in Steps 3-5.

To conclude this subsection, we present our row generation procedure in Algorithm 2.




In Algorithm 2, we first initialize the set $U=\varnothing$ in Step 1. We then solve problem (27) in Step 2 using the dual simplex method with the warm start information in the last step, see for example [21]. In Step 3, the optimal value of problem $(27)$ is nonpositive, and hence point $(\bar{x}, \bar{y}) \in P(\bar{x}, \bar{y})$. In Step 4 , we modify the code BOUKNAP, written by Pisinger [27], to solve the unbounded integer knapsack problem (25) with fractional value costs. In Step 5, we know the current inequality is invalid for $P(\bar{x}, \bar{y})$. Therefore, we first modify the solution (obtained in Step 4) using Algorithm 1 such that it corresponds to a (possibly) stronger inequality. Then we add the corresponding inequality to problem (27) and go to Step 2. Finally, in Step 6, if $\tilde{z} \leq 0$, we obtain the valid inequality $(24)$, which is violated by point $(\bar{x}, \bar{y})$.

\subsection{Numerical errors}

Due to the numerical errors incurred in solving problem (27), we may get an invalid inequality in Algorithm 2. To avoid this, we scale the inequality to obtain integral coefficients by solving the integer programming problem

$$
\begin{aligned}
\min _{\alpha, \beta, \theta} & \theta, \\
\text { s.t. } & \bar{\beta} \theta=\beta, \\
& \bar{\alpha} \theta=\alpha, \\
& \beta_{t} \in \mathbb{Z}, \quad \forall t \in \bar{T}, \\
& \alpha_{q} \in \mathbb{Z}, \forall q \in \bar{Q}, \\
& \theta \geq 1 .
\end{aligned}
$$

Vasilyev et al. [31] suggested to use the enumeration of the multiplier $\theta$ from 2 to $10^{4}$ and checked the integrality within the tolerance $10^{-5}$. To further avoid too much computational efforts, here we use the approach described in [1]. More formally, let $\frac{\lambda_{q}}{\mu_{q}}$ be the rational representation of coefficient $\bar{\alpha}_{q}$. Note that $\frac{\lambda_{q}}{\mu_{q}}$ can be obtained by using the Euclidean algorithm within a small tolerance. To avoid too large numbers, we give the following requirements:

$$
\left|\frac{\lambda_{q}}{\mu_{q}}-\bar{\alpha}_{q}\right| \leq \epsilon:=10^{-9},\left|\lambda_{q}\right| \leq \lambda_{\max }:=10^{6} \text {, and } \mu_{q} \leq \mu_{\max }:=10^{3}
$$

The representation of $\bar{\beta}_{t}$ for each $t \in T$ is also required to be satisfied with the same restrictions. Let $\mu$ be the least common multiple of all denominators. We also require $\mu \leq \mu_{\max }$. We scale $\bar{\alpha}$ and $\bar{\beta}$ by setting $\alpha_{q}=\mu \bar{\alpha}_{q}$ for all $q \in \bar{Q}$ and $\beta_{t}=\mu \bar{\beta}_{t}$ for all $t \in \bar{T}$ with the requirement that $\left|\mu \alpha_{q}\right| \leq \mu_{\max }$ and $\left|\mu \beta_{t}\right| \leq \mu_{\max }$. If all the requirements are satisfied, we accept this inequality; otherwise, we drop it. 
After scaling $\alpha_{q}$ for all $q \in Q$ and $\beta_{t}$ for all $t \in T$, we recompute the right hand side $\gamma$ by solving the unbounded integer knapsack problem

$$
\gamma=\max _{x, y}\left\{\sum_{q \in \bar{Q}} \alpha_{q} x_{q}-\sum_{t \in \bar{T}} \beta_{t} y_{t}:(x, y) \in X(\bar{x}, \bar{y})\right\} .
$$

This leads to the inequality

$$
\sum_{q \in \bar{Q}} \alpha_{q} x_{q} \leq \sum_{t \in \bar{T}} \beta_{t} y_{t}+\gamma
$$

which is valid for $P(\bar{x}, \bar{y})$.

\subsection{Sequential lifting}

The inequality (33) is valid for $P(\bar{x}, \bar{y})$. However, in general, it may be invalid for $P$. To resolve this problem, the variables, which are fixed in the preprocessing step, are sequentially lifted according to a given lifting order $\Pi$, i.e., a permutation of $(Q \backslash \bar{Q}) \cup(T \backslash \bar{T})$. We now illustrate the procedure to lift the first variable with index $k$ based on inequality (33). Denote

$$
W(C)=\max _{(x, y) \in\{0,1\}|\bar{Q}| \times \mathbb{Z}_{+}^{|\bar{T}|}}\left\{\sum_{q \in \bar{Q}} \alpha_{q} x_{q}-\sum_{t \in \bar{T}} \beta_{t} y_{t}: \sum_{q \in \bar{Q}} a_{q} x_{q} \leq \sum_{t \in \bar{T}} b_{t} y_{t}+C\right\} .
$$

We have the following three cases.

1) If $k \in Q \backslash \bar{Q}$ and $\bar{x}_{k}=0$, then the lifted inequality is

$$
\sum_{q \in \bar{Q}} \alpha_{q} x_{q}+\alpha_{k} x_{k} \leq \sum_{t \in \bar{T}} \beta_{t} y_{t}+\gamma,
$$

where $\alpha_{k}=\gamma-W\left(\bar{c}-a_{k}\right)$.

2) If $k \in Q \backslash \bar{Q}$ and $\bar{x}_{k}=1$, then the lifted inequality is

$$
\sum_{q \in \bar{Q}} \alpha_{q} x_{q}+\alpha_{k}\left(x_{k}-1\right) \leq \sum_{t \in \bar{T}} \beta_{t} y_{t}+\gamma,
$$

where $\alpha_{k}=W\left(\bar{c}+a_{k}\right)-\gamma$.

3) If $k \in T \backslash \bar{T}$ with $\bar{y}_{k}=0$, then the lifted inequality is

$$
\sum_{q \in \bar{Q}} \alpha_{q} x_{q} \leq \sum_{t \in \bar{T}} \beta_{t} y_{t}+\beta_{k} y_{k}+\gamma,
$$

where

$$
\beta_{k}=\max \left\{\left(W\left(\bar{c}+\ell b_{k}\right)-\gamma\right) / \ell: \ell=1, \ldots, \bar{\ell}\right\} .
$$

Here we set $\bar{\ell}=\left\lceil\left(\sum_{q \in \bar{Q}} a_{q}-\bar{c}\right) / b_{k}\right\rceil$ since $W\left(\bar{c}+\ell b_{k}\right)=\sum_{q \in \bar{Q}} \alpha_{q}$ for all $\ell \geq \bar{\ell}$. 
Similarly, we may continue to lift the other variables, which are fixed in the preprocessing step in Sect. 4.1, to obtain a valid inequality of polyhedron $P$. During the whole lifting process, we need to solve several integer knapsack problems. This can be done via the dynamic programming algorithm. For more details, we refer to [31].

\section{Numerical results}

In order to test the effectiveness of the exact separation algorithm for solving the unsplittable capacitated network design problem, we implement it in $\mathrm{C}++$ linked with IBM ILOG CPLEX optimizer 12.7.1 [16] library. Following [31], to avoid changing the problem structure, the presolving features are turned off in our experiments. Moreover, the dual simplex method is used to reoptimize the linear programming problem (27) after adding cutting planes. To eliminate the effect of multithreads, the computations are implemented in a single thread. The time limit is set to 7200 seconds. Except where explicitly stated, the other parameters in CPLEX are set to the default ones. The exact separation procedure stops if the optimal value of the LP relaxation problem of the unsplittable capacitated network design problem improves by less than $0.01 \%$ between two adjacent calls.

\subsection{Testsets}

We conduct our computational study on three testsets of the unsplittable capacitated network design problem (1)-(4). The first testset NDP1, studied in Atamtürk et al. [4], contains 20 instances with a single facility. We use this testset to compare the performance effect of the cuts constructed in our exact separation procedure with that of existing cuts studied in the literature. The second testset NDP2 includes 26 realistic network instances generated by the Survivable Network Design Library (SNDlib 1.0) [26]. 9 of them are instances with a single facility and 17 of them are instances with multifacility. To possibly reduce the unstable behavior of integer programming solvers (see, e.g., $[18,22]$ ), we solve each instance using 10 different random seeds in 7200 seconds. We treat every pair of instance and seed as an individual model, which results in 200 models for testset NDP1 and 260 models for testset NDP2 .

The third testset NDP3 is randomly generated based on [23]. We use this testset to evaluate the performance effects of different capacity module sizes and module costs on the unsplittable capacitated network design problem using standard integer programming solver or our exact separation procedure. Table 1 lists different capacity module sizes and module costs studied in [23]. For the same facility, we assume that its module costs on all arcs are the same. In total, we study problems with 27 different capacities and costs structures. We generate the underlying graphs with 50 vertices using the procedure described in [23]; see also [24,29]. We generate 10 graphs with 20 commodities 
with random source nodes and destination nodes. The demand of each commodity is chosen uniformly in $\{10,11, \ldots, 190\}$. For each graph, 27 problems are generated based on each item of capacity module sizes and costs in Table 1. Thus, in total, we have 270 models for testset NDP3.

Table 1: Capacity module sizes and module costs of testset NDP3.

\begin{tabular}{|l|l|l|}
\hline & Capacity module sizes & Capacity module costs \\
\hline 1_1_1 & $(130)$ & $(10000)$ \\
2_1_1 & $(130,50)$ & $(10000,5000)$ \\
3_1_1 & $(130,50,20)$ & $(10000,5000,2500)$ \\
\hline 1_1_2 & $(130)$ & $(18000)$ \\
2_1_2 & $(130,50)$ & $(18000,9000)$ \\
3_1_2 & $(130,50,20)$ & $(18000,9000,5000)$ \\
\hline 1_1_3 & $(130)$ & $(25000)$ \\
2_1_3 & $(130,50)$ & $(25000,13000)$ \\
3_1_3 & $(130,50,20)$ & $(25000,13000,9000)$ \\
\hline 1_2_1 & $(170)$ & $(10000)$ \\
2_2_1 & $(170,70)$ & $(10000,5000)$ \\
3_2_1 & $(170,70,30)$ & $(10000,5000,2500)$ \\
\hline 1_2_2 & $(170)$ & $(18000)$ \\
2_2_2 & $(170,70)$ & $(18000,9000)$ \\
3_2_2 & $(170,70,30)$ & $(18000,9000,5000)$ \\
\hline 1_2_3 & $(170)$ & $(25000)$ \\
2_2_3 & $(170,70)$ & $(25000,13000)$ \\
3_2_3 & $(170,70,30)$ & $(25000,13000,9000)$ \\
\hline 1_3_1 & $(200)$ & $(10000)$ \\
2_3_1 & $(200,80)$ & $(10000,5000)$ \\
3_3_1 & $(200,80,30)$ & $(10000,5000,2500)$ \\
\hline 1_3_2 & $(200)$ & $(18000)$ \\
2_3_2 & $(200,80)$ & $(18000,9000)$ \\
3_3_2 & $(200,80,30)$ & $(18000,9000,5000)$ \\
\hline 1_3_3 & $(170)$ & $(25000)$ \\
2_3_3 & $(200,80)$ & \\
3_3_3 & $(200,80,30)$ & $(25000,13000)$ \\
\hline
\end{tabular}

\subsection{Different lifting orders}

In the sequential lifting step, different lifting orders lead to different inequalities [31]. Hence the first experiment is conducted to test performance effect of different lifting orders. We consider the following four lifting orders.

- LIFT1: Variables, fixed to one, are lifted first in decreasing order of their coefficients. Then variables, fixed to zero, are lifted also in decreasing order of their coefficients. 
- LIFT2: Variables are lifted in decreasing order of their coefficients.

- LIFT3: Variables are lifted in decreasing order of their reduced costs.

- LIFT4: Variables are lifted in increasing order of their reduced costs.

Table 2: Performance comparison of different lifting orders.

\begin{tabular}{|l|cccc|}
\hline Testset & LIFT1 & LIFT2 & LIFT3 & LIFT4 \\
\hline NDP1 & 71.51 & 71.51 & 71.52 & 71.54 \\
NDP2 & 54.39 & 54.49 & 54.12 & 54.96 \\
NDP3 & 87.35 & 87.29 & 87.21 & 87.40 \\
\hline
\end{tabular}

Table 2 presents the arithmetic means of the gap closeds [33] of all models in the corresponding testsets. The gap closed is defined as

$$
100 \cdot \frac{z_{\mathrm{root}}-z_{\mathrm{LP}}}{z_{\mathrm{ub}}-z_{\mathrm{LP}}}
$$

where $z_{\text {root }}$ is the objective value of the LP relaxation at root node after adding cuts, $z_{\mathrm{LP}}$ is the value of the LP relaxation before adding cuts, and $z_{\mathrm{ub}}$ is the value of the best known value of the model. Table 2 shows that using the lifting order LIFT4, the gap closed is slightly better than those of other lifting orders in all the three testsets. Therefore, in further computational studies, the lifting order LIFT4 is used in our exact separation algorithm.

\subsection{Performance effect of the exact separation procedure}

In this subsection, we evaluate the performance effect of adding cuts generated by the exact separation algorithm into the solver.

Table 3: Performance comparison of the exact separation procedure with the default setting for testset NDP1.

\begin{tabular}{|l|c|ccc|ccccc|}
\hline \multirow{2}{*}{ Bracket } & \multirow{2}{*}{ total } & \multicolumn{3}{|c|}{ CPX } & \multicolumn{5}{c|}{ EXACT } \\
\cline { 3 - 10 } & & solved & nodes & time & solved & nodes & time & faster & slower \\
\hline all & 200 & 145 & 1313 & 32 & 151 & 1105 & 31 & 34 & 79 \\
{$[1,7200]$} & 125 & 116 & 2968 & 48 & 122 & 2476 & 46 & 34 & 65 \\
{$[10,7200]$} & 61 & 52 & 33410 & 304 & 58 & 29352 & 274 & 23 & 21 \\
{$[100,7200]$} & 34 & 25 & 296061 & 2266 & 31 & 219844 & 1733 & 21 & 5 \\
{$[1000,7200]$} & 24 & 15 & 423246 & 4142 & 21 & 356030 & 3469 & 13 & 4 \\
\hline
\end{tabular}

Tables 3-5 compare the computational results obtained by using CPLEX $(\mathrm{CPX})$ and our exact separation procedure (EXACT). We report the number of solved models, the average running time, and the average number of explored nodes ${ }^{1}$. Besides, columns "faster" and "slower" report the number of

\footnotetext{
1 Shifted geometric mean, 10s for average time and 100 for average nodes [1].
} 
Table 4: Performance comparison of the exact separation procedure with the default setting for testset NDP2.

\begin{tabular}{|l|c|ccc|ccccc|}
\hline \multirow{2}{*}{ Bracket } & \multirow{2}{*}{ total } & \multicolumn{3}{|c|}{ CPX } & \multicolumn{5}{c|}{ EXACT } \\
\cline { 3 - 10 } & & solved & nodes & time & solved & nodes & time & faster & slower \\
\hline all & 260 & 55 & 26062 & 291 & 63 & 21904 & 269 & 33 & 20 \\
{$[1,7200]$} & 63 & 55 & 26062 & 291 & 63 & 21904 & 269 & 33 & 20 \\
{$[10,7200]$} & 56 & 48 & 38772 & 427 & 56 & 31884 & 391 & 30 & 17 \\
{$[100,7200]$} & 49 & 41 & 48949 & 595 & 49 & 38996 & 531 & 27 & 14 \\
{$[1000,7200]$} & 19 & 11 & 113188 & 3410 & 19 & 86696 & 2738 & 12 & 6 \\
\hline
\end{tabular}

Table 5: Performance comparison of the exact separation procedure with the default setting for testset NDP3.

\begin{tabular}{|l|c|ccc|ccccc|}
\hline \multirow{2}{*}{ Bracket } & \multirow{2}{*}{ total } & \multicolumn{3}{|c|}{ CPX } & \multicolumn{5}{c|}{ EXACT } \\
\cline { 3 - 9 } & & solved & nodes & time & solved & nodes & time & faster & slower \\
\hline all & 270 & 139 & 165616 & 1180 & 210 & 9172 & 145 & 196 & 7 \\
{$[1,7200]$} & 210 & 139 & 165616 & 1180 & 210 & 9172 & 145 & 196 & 7 \\
{$[10,7200]$} & 203 & 132 & 202814 & 1374 & 203 & 10758 & 158 & 193 & 4 \\
{$[100,7200]$} & 178 & 107 & 331671 & 2211 & 178 & 16789 & 212 & 173 & 1 \\
{$[1000,7200]$} & 125 & 54 & 704511 & 4781 & 125 & 31822 & 359 & 120 & 1 \\
\hline
\end{tabular}

models that get at least $10 \%$ faster and slower, respectively. We group the three testsets into several brackets. The bracket "all" contains the models which can be solved by at least one of the settings. The bracket $[n, 7200]$ contains the models which can be solved by the slower setting in at least $n$ seconds. The larger $n$ is, the harder of the model is. For each bracket, we report the number of considered models in column "total".

As it can be observed in Tables 3-5, our exact separation algorithm has a positive effect on all these three testsets, especially on the hard models. In particular, using the exact separation algorithm, we can solve 6,8 , and 71 more models than those using the default setting on testsets NDP1, NDP2, and NDP3, respectively. This clearly shows that our exact separation algorithm can improve the performance of the solver on solving the unsplittable capacitated network design problem.

Specifically, for testset NDP1, using the exact separation algorithm, we have 79 models for which the running times are slower than that of the default setting while only 34 models are solved faster. This is due to the fact that these models are easier than models in testsets NDP2 and NDP3, and, as a result, the benefit of the exact separation algorithm cannot compensate for its additional overhead on these easy models.

For testset NDP2, we notice that these models are harder than those in testset NDP1 or NDP3. Among these 260 models, CPX and EXACT only solve 55 and 63 of them, respectively. Nevertheless, for the solved models, using EXACT, the average running time decreases from 291s to 269s.

For testset NDP3, Table 5 shows a significant improvement of using our exact separation algorithm. The average running time decreases from 1180s 
Table 6: Results of different capacity module sizes and module costs on testset NDP3.

\begin{tabular}{|c|ccc|ccc|}
\hline & \multicolumn{3}{|c|}{ CPX } & \multicolumn{3}{|c|}{ EXACT } \\
\cline { 2 - 7 } & nodes & time & solved & nodes & time & solved \\
\hline 1_1_1 & 101498 & 1107 & 6 & 52605 & 744 & 7 \\
2_1_1 & 133392 & 833 & 7 & 6596 & 96 & 9 \\
3_1_1 & 757202 & 2451 & 5 & 2857 & 55 & 9 \\
\hline 1_1_2 & 115713 & 1274 & 6 & 48061 & 710 & 7 \\
2_1_2 & 120158 & 794 & 7 & 6767 & 101 & 8 \\
3_1_2 & 361194 & 1367 & 6 & 2927 & 66 & 9 \\
\hline 1_1_3 & 98817 & 1081 & 6 & 50658 & 709 & 7 \\
2_1_3 & 135529 & 892 & 6 & 7409 & 99 & 8 \\
3_1_3 & 280378 & 1178 & 7 & 4800 & 78 & 10 \\
\hline 1_2_1 & 77609 & 1228 & 6 & 44647 & 987 & 6 \\
2_2_1 & 221946 & 1313 & 6 & 21803 & 217 & 9 \\
3_2_1 & 364549 & 1700 & 5 & 4373 & 43 & 10 \\
\hline 1_2_2 & 82223 & 1270 & 6 & 44890 & 973 & 6 \\
2_2_2 & 157895 & 1096 & 6 & 22698 & 242 & 8 \\
3_2_2 & 487028 & 2328 & 5 & 4140 & 47 & 10 \\
\hline 1_2_3 & 87375 & 1373 & 5 & 42778 & 889 & 6 \\
2_2_3 & 165320 & 1115 & 6 & 21347 & 244 & 9 \\
3_2_3 & 321156 & 1579 & 8 & 16831 & 149 & 10 \\
\hline 1_3_1 & 247636 & 4362 & 4 & 134158 & 3010 & 7 \\
2_3_1 & 334434 & 3353 & 3 & 77329 & 1019 & 7 \\
3_3_1 & 507180 & 3190 & 4 & 14150 & 165 & 10 \\
\hline 1_3_2 & 223731 & 3916 & 3 & 125455 & 2897 & 5 \\
2_3_2 & 283211 & 2889 & 3 & 90339 & 1325 & 6 \\
3_3_2 & 536980 & 3464 & 3 & 14477 & 199 & 9 \\
\hline 1_3_3 & 231183 & 4150 & 3 & 144692 & 3252 & 5 \\
2_3_3 & 285635 & 2936 & 3 & 122664 & 1513 & 5 \\
3_3_3 & 338583 & 2674 & 4 & 33673 & 460 & 8 \\
\hline
\end{tabular}

to $145 \mathrm{~s}$. To further see where the improvement comes from, we report the computational results of different capacity module sizes and costs of facilities independently in Table 6 . To be more specific, we list the results in 9 different groups corresponding to Table 1 . In each group, the number of facilities is different in each item (i.e. $i_{-} j_{-} k$ ). There are 10 different models in each item with different network structures and commodities. We report the number of average nodes, the average running time, and the number of solved models in each item. In the same group, we can observe that with our exact separation algorithm, the models with more facilities are easier to be solved than those with fewer facilities. However, the same behavior cannot be observed in the computational results of CPX. This shows that compared with problems with a single facility, our exact separation algorithm works better in problems with more facilities. 


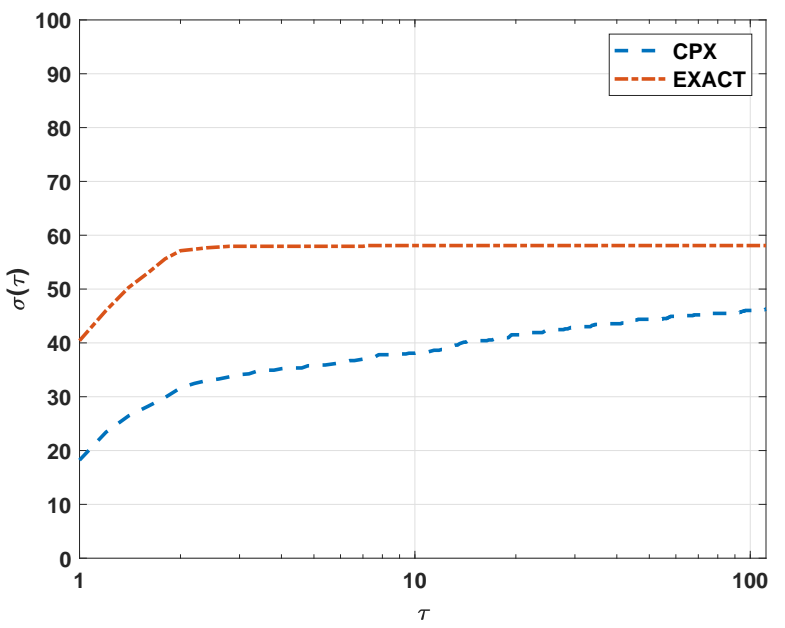

Fig. 1: Performance profile for testsets NDP1, NDP2, and NDP3.

To end of this subsection, we plot the performance profiles of CPX and EXACT in Fig. 1 to further compare their performance. For each model in the testsets NDP1, NDP2, and NDP3, we compute a factor $\tau$ as the ratio of the running time to solve to optimality of the considered setting to the minimum running time of two settings CPX and EXACT. Each point $(\tau, \sigma)$ of the curves in Fig. 1 represents that in $\sigma$ percentage of the models, this particular setting is at most $\tau$ times slower than the faster setting; for more details, see [17]. Consequently, the higher the curve is, the better the setting performs. Fig. 1 clearly shows that EXACT performs much better than CPX. In particular, EXACT is able to solve $58.1 \%$ of the models to optimality while CPX is only able to solve $46.3 \%$ of the models to optimality.

\subsection{Comparison with c-strong inequality $[13,14]$}

According to [3], the c-strong inequality $[13,14]$ is quite effective in solving the unsplittable capacitated network design problem. Other proposed inequalities $[4,30]$ can provide additional improvement but the marginal effect on top of the c-strong inequality is limited. Therefore, we only compare the performance effects of the inequality generated by the exact separation procedure (EXACT) with the c-strong inequality (CSTRONG). Since the c-strong inequality can only be applied in the case of one facility or two facilities with divisible capacities, we only conduct experiments on testset NDP1. The results are reported in Table 7.

Table 7 shows that the performance of EXACT is better than that of CSTRONG, especially on the hard models. In total, EXACT solves 4 more models than CSTRONG. This shows that even compared with the existing 
Table 7: Performance comparison with the c-strong inequalities on testset NDP1.

\begin{tabular}{|l|c|ccc|ccccc|}
\hline \multirow{2}{*}{ Bracket } & \multirow{2}{*}{ total } & \multicolumn{3}{|c|}{ CSTRONG } & \multicolumn{5}{c|}{ EXACT } \\
\cline { 3 - 10 } & & solved & nodes & time & solved & nodes & time & faster & slower \\
\hline all & 200 & 147 & 1232 & 31 & 151 & 1033 & 28 & 53 & 67 \\
{$[1,7200]$} & 130 & 125 & 2281 & 41 & 129 & 1883 & 38 & 45 & 62 \\
{$[10,7200]$} & 60 & 55 & 31508 & 275 & 59 & 25637 & 232 & 27 & 22 \\
{$[100,7200]$} & 32 & 27 & 366689 & 2374 & 31 & 222227 & 1585 & 20 & 4 \\
{$[1000,7200]$} & 22 & 17 & 579321 & 4725 & 21 & 377860 & 3246 & 13 & 3 \\
\hline
\end{tabular}

polyhedral studies on the unsplittable flow arc-set polyhedron, our exact separation algorithm is more effective in solving the unsplittable capacitated network design problem.

\subsection{Performance effect of using Propositions 5-8}

We now report the performance effect of Propositions 5-8 in our procedure in Table 8. The average results over all the models in each testset are presented. Compared with EXACT, NOPROS refers to the setting of calling the row generation subroutine to solve the separation problem (21) even if one of the conditions in Propositions 5-8 is satisfied. For each setting, we report the running time spent in the row generation subroutine in column "rgtime". For EXACT, we additionally report the numbers of times that fulfilling the conditions in Propositions 5-8 in "p5"- "p8", respectively. In column "ncalls", we report the number of calling the exact separation algorithm. In column "rate", we list the successful rate computed by the number of times fulfilling at least one of the conditions in Propositions 5-8 over the number of calling the exact separation algorithm.

Table 8: Performance effect of using Propositions 5-8.

\begin{tabular}{|c|c|ccccccc|}
\hline \multirow{2}{*}{ Testset } & NOPROS & \multicolumn{6}{|c|}{ EXACT } \\
\cline { 2 - 9 } & rgtime & rgtime & p5 & p6 & p7 & p8 & ncalls & rate \\
\hline NDP1 & 1.21 & 0.80 & 255 & 543 & 0 & 0 & 1158 & $66.42 \%$ \\
NDP2 & 102.91 & 91.10 & 183 & 151 & 10 & 411 & 1361 & $31.72 \%$ \\
NDP3 & 2.90 & 2.57 & 425 & 1020 & 5 & 19 & 3182 & $46.17 \%$ \\
\hline
\end{tabular}

As it can be seen in Table 8, using Propositions 5-8, the running time of the separation algorithm decreases considerably. This is due to the fact that, among the total number of the calls of the exact separation algorithm, $66.42 \%$, $31.72 \%$, and $46.17 \%$ of them can be computed directly using Propositions 5-8 for testsets NDP1, NDP2, and NDP3 without calling the time-consuming row generation subroutine. Since the models in testset NDP1 contain only a single facility, the conditions in Propositions 5 and 6 occur frequently while those 
in Propositions 7 and 8 never occur. Compared with those in Propositions 5 and 6, the conditions in Propositions 7 and 8 occur less frequently. This is not surprising since the conditions in Propositions 7 and 8 are much stricter than those in Propositions 5 and 6. Finally, we observe that the reduction on the running time is not consistent with that of the successful rate. This is because, using the row generation subroutine, the separation problems that fulfill one of the conditions in Propositions 5-8 are easier to be solved than those that do not fulfill any of them.

\subsection{Performance effect of using Algorithm 1}

Finally, we report the performance effect of employing Algorithm 1 in the exact separation procedure. Compared with EXACT, NOALG2 refers to the setting of the exact separation procedure without using Algorithm 1. Notice that this may lead to a weaker constraint (30) in the row generation subroutine. For each setting, we report the running time of the row generation subroutine and the average iteration of a row generation call in columns "rgtime" and "iter", respectively. We present the average results among all the models.

Table 9: Performance effect of using Algorithm 1.

\begin{tabular}{|c|cc|cc|}
\hline \multirow{2}{*}{ Testset } & \multicolumn{2}{|c|}{ NOALG2 } & \multicolumn{2}{c|}{ EXACT } \\
\cline { 2 - 5 } & rgtime & iter & rgtime & iter \\
\hline NDP1 & 1.42 & 8.87 & 0.80 & 4.63 \\
NDP2 & 144.20 & 61.62 & 91.10 & 21.83 \\
NDP3 & 3.92 & 9.22 & 2.57 & 6.36 \\
\hline
\end{tabular}

As it can be seen in Table 9, with Algorithm 1, the iteration of row generation subroutine per call decreases significantly, which in turn, reduces $43.66 \%$, $36.77 \%$, and $34.44 \%$ of the row generation time in testsets NDP1, NDP2, and NDP3, respectively. This confirms that our proposed algorithm indeed works well in practice. Notice that it is reasonable to observe reductions on the average iteration exceeds reduction on the running time since Algorithm 1 also leads to a denser constraint for the linear programming problem (27).

\section{Conclusion and future work}

In this paper, we have considered the separation problem of the flow arcset polyhedron in the unsplittable capacitated network design problem. By solving the separation problem, we generated the facet-defining inequality for the considered polyhedron. We showed that in some special cases, a closed form of the separation problem can be derived. For the general case, we used the exact separation algorithm to solve the separation problem. Moreover, a new technique was proposed to reduce the computational time in the row 
generation subroutine of the exact separation algorithm. The numerical experiments showed the effectiveness of the exact separation algorithm in solving the unsplittable capacitated network design problem and the advantage of the proposed technique in reducing the exact separation time.

There still exist some ideas to be explored in this study. For the flow arc-set polyhedron, we have proposed a new technique to speed up the row generation subroutine; see Algorithm 1. It deserves to test whether or not the same improvement can be observed for the knapsack polyhedron. In this study, we only implemented the exact separation algorithm on the flow arc-set polyhedron, but it can be extended on the cut-set polyhedron, see for example [2]. We are currently conducting this topic to see the performance effect of generalizing this to the cut-set polyhedron.

\section{References}

1. Achterberg, T.: SCIP: solving constraint integer programs. Mathematical Programming Computation 1, 1-41 (2009)

2. Achterberg, T., Raack, C.: The MCF-separator: detecting and exploiting multicommodity flow structures in MIPs. Mathematical Programming Computation 2, 125$165(2010)$

3. Atamturk, A., Gunluk, O.: Multi-commodity multi-facility network design. arXiv preprint arXiv:1707.03810 (2017)

4. Atamtürk, A., Rajan, D.: On splittable and unsplittable flow capacitated network design arc-set polyhedra. Mathematical Programming 92, 315-333 (2002)

5. Avella, P., Boccia, M., Mattia, S.: A branch-and-cut algorithm for the single source capacitated facility location problem. In: 2013 International Conference on Advanced Logistics and Transport, pp. 181-186 (2013)

6. Avella, P., Boccia, M., Vasilyev, I.: A computational study of exact knapsack separation for the generalized assignment problem. Computational Optimization and Applications 45, 543-555 (2010)

7. Barnhart, C., Hane, C.A., Vance, P.H.: Using branch-and-price-and-cut to solve origindestination integer multicommodity flow problems. Operations Research 48(2), 318-326 (2000)

8. Benhamiche, A., Mahjoub, A.R., Perrot, N., Uchoa, E.: Unsplittable non-additive capacitated network design using set functions polyhedra. Computers \& Operations Research 66, 105-115 (2016)

9. Boccia, M., Hanafi, S., Vasilyev, I.: New computational results with an exact knapsack separation procedure for structured binary integer programming problems. In: 2013 5th International Conference on Modeling, Simulation and Applied Optimization (ICMSAO), pp. 1-5 (2013)

10. Boyd, E.A.: Generating fenchel cutting planes for knapsack polyhedra. SIAM Journal on Optimization 3(4), 734-750 (1993)

11. Boyd, E.A.: Fenchel cutting planes for integer programs. Operations Research 42(1), 53-64 (1994)

12. Boyd, E.A.: On the convergence of fenchel cutting planes in mixed-integer programming. SIAM Journal on Optimization 5(2), 421-435 (1995)

13. Brockmüller, B., Günlück, O., Wolsey, L.A.: Designing private line networks - Polyhedral analysis and computation. CORE Discussion Papers 1996047, Université catholique de Louvain (1996)

14. Brockmüller, B., Günlück, O., Wolsey, L.A.: Designing private line networks. Transactions on Operational Research 16(1-2), 7-24 (2004)

15. Chopra, S., Gilboa, I., Sastry, S.: Source sink flows with capacity installation in batches. Discrete Applied Mathematics 85(3), 165-192 (1998) 
16. CPLEX: https://www.ibm.com/analytics/cplex-optimizer

17. Dolan, E.D., Moré, J.J.: Benchmarking optimization software with performance profiles. Mathematical Programming 91, 201-213 (2002)

18. Fischetti, M., Monaci, M.: Exploiting erraticism in search. Operations Research 62(1), 114-122 (2014)

19. Gavish, B., Altinkemer, K.: Backbone network design tools with economic tradeoffs. ORSA Journal on Computing 2(3), 236-252 (1990)

20. Kaparis, K., Letchford, A.N.: Separation algorithms for 0-1 knapsack polytopes. Mathematical Programming 124, 69-91 (2010)

21. Koberstein, A.: The dual simplex method, techniques for a fast and stable implementation. Ph.D. thesis, Universität Paderborn (2005)

22. Lodi, A., Tramontani, A.: Performance variability in mixed-integer programming. In: Topaloglu, H. (ed.) TutORials in Operations Research: Theory Driven by Influential Applications, INFORMS, Catonsville, 1-12 (2013)

23. Luo, H., Kianfar, K.: n-step cutset inequalities: facets for multi-module capacitated network design problem (2019)

24. Magnanti, T.L., Mirchandani, P., Vachani, R.: Modeling and solving the two-facility capacitated network loading problem. Operations Research 43(1), 142-157 (1995)

25. Minkowski, H.H.: Geometrie der Zahlen. Teubner-Verlag, Stuttgart (1896)

26. Orlowski, S., Wessäly, R., Pióro, M., Tomaszewski, A.: SNDlib 1.0-Survivable Network Design Library. Networks 55(3), 276-286 (2010)

27. Pisinger, D.: A minimal algorithm for the bounded knapsack problem. INFORMS Journal on Computing 12(1), 75-82 (2000)

28. Raack, C., Koster, A.M., Orlowski, S., Wessäly, R.: On cut-based inequalities for capacitated network design polyhedra. Networks 57(2), 141-156 (2011)

29. Salman, F., Ravi, R., Hooker, J.: Solving the capacitated local access network design problem. INFORMS Journal on Computing 20(2), 243-254 (2008)

30. Van Hoesel, S.P., Koster, A.M., van de Leensel, R.L., Savelsbergh, M.W.: Polyhedral results for the edge capacity polytope. Mathematical Programming 92, 335-358 (2002)

31. Vasilyev, I., Boccia, M., Hanafi, S.: An implementation of exact knapsack separation. Journal of Global Optimization 66, 127-150 (2016)

32. Weyl, H.: The elementary of convex polyhedra. In: Contributions to the Theory of Games, H.W. Kuhn and A.W. Tucker, eds.,. Princeton University Press, Princeton, 3-18 (1952)

33. Wolter, K.: Implementation of cutting plane separators for mixed integer programs. Dipolma thesis, Technische Universität Berlin (2006) 
Appendix

Lemma 2 Suppose that $T=\{1\}$ and inequality (6) with $\beta_{1}=1$ is a facetdefining inequality of polyhedron $P$. For each $q \in Q$, if $a_{q} \leq b_{1}$, then $0 \leq \alpha_{q} \leq$ 1 .

Proof Combining with $a_{q} \leq b_{1}, \beta_{1}=1$, and Proposition 4, we have the statement.

Lemma 3 Given $\left(\bar{x}, \bar{y}_{1}\right) \in[0,1]^{|Q|} \times \mathbb{R}_{+}$, the linear programming problem

$$
\begin{aligned}
\max _{\alpha, \gamma}\left\{\sum_{q \in Q} \bar{x}_{q} \alpha_{q}-\bar{y}_{1}-\gamma:-r-\gamma \leq 0,\right. & \\
& \left.\sum_{q \in Q} \alpha_{q}-(r+1)-\gamma \leq 0,0 \leq \alpha_{q} \leq 1, \forall q \in Q\right\}
\end{aligned}
$$

has an optimal solution $\left(\boldsymbol{e}^{\boldsymbol{d}},-r\right)$ where $r$ and $d$ are defined in (16) and (17), respectively.

Proof By simple calculation, point $\left(\boldsymbol{e}^{\boldsymbol{d}},-r\right)$ is a feasible solution of problem (37) with the objective value being $\bar{x}_{d}-\bar{y}_{1}+r$. It is optimal since

$$
\begin{aligned}
& \sum_{q \in Q} \bar{x}_{q} \alpha_{q}-\bar{y}_{1}-\gamma \\
\leq & \bar{x}_{d} \sum_{q \in Q} \alpha_{q}-\bar{y}_{1}-\gamma \quad \\
\leq & \bar{x}_{d}[\gamma+(r+1)]-\bar{y}_{1}-\gamma \quad\left(\text { from } \alpha_{q} \geq 0 \text { and the definition of } d \text { in }(17)\right) \\
= & \left.\bar{x}_{d}+\bar{x}_{d} r-\left(1-\bar{x}_{d}\right) \gamma-\bar{y}_{1}-(r+1)-\gamma \leq 0 \text { in problem }(37)\right) \\
\leq & \bar{x}_{d}+\bar{x}_{d} r+\left(1-\bar{x}_{d}\right) r-\bar{y}_{1} \\
= & \bar{x}_{d}-\bar{y}_{1}+r .
\end{aligned}
$$

\section{Proof of Proposition 5}

Proof From the definition of $r$ in (16), we have $(\mathbf{0}, r) \in X$. This, combined with assumption (ii) and condition (18), implies that

$$
X=\{(\mathbf{0}, r)\} \cup\left\{(x, k) \in\{0,1\}^{|Q|} \times \mathbb{Z}_{+}: k \geq r+1\right\} .
$$

It is obvious that the vertices of polyhedron $P$, i.e., conv $(X)$, are points $(\mathbf{0}, r)$ and $(x, r+1)$ for all $x \in\{0,1\}^{|Q|}$ with $x \neq \mathbf{0}$. Particularly, in problem (12), the vertex $(\boldsymbol{e}, r+1)$ corresponds to the constraint

$$
\sum_{q \in Q} \alpha_{q}-(r+1)-\gamma \leq 0
$$


By removing the constraints that are dominated by (38) and using Lemma 2 and Theorem 1, problem (12) is further equivalent to problem (37). Hence, by Lemma 3 , in this case, point $\left(\boldsymbol{e}^{d},-r\right)$ is optimal for problem (12), which corresponds to the inequality $x_{d} \leq y_{1}-r$ of polyhedron $P$. Furthermore, inequality $x_{d} \leq y_{1}-r$ is facet-defining for polyhedron $P$ since the $|Q|+1$ affinely independent points $(\mathbf{0}, r),\left(\boldsymbol{e}^{\boldsymbol{d}}, r+1\right)$, and $\left(\boldsymbol{e}^{\boldsymbol{d}}+\boldsymbol{e}^{\boldsymbol{q}}, r+1\right)$ for each $q \in Q \backslash\{d\}$ are on the face $\left\{\left(x, y_{1}\right) \in P: x_{d}=y_{1}-r\right\}$.

Lemma 4 Let $r$ and $d$ be the values defined in (16) and (17), respectively, and $\left(\bar{x}, \bar{y}_{1}\right) \in[0,1]^{|Q|} \times \mathbb{R}_{+}$. Consider the following linear programming problem

$$
\begin{aligned}
\max _{\alpha, \gamma}\left\{\sum_{q \in Q} \bar{x}_{q} \alpha_{q}-\bar{y}_{1}-\gamma:-r-\gamma \leq 0,\right. \\
\\
\left.\sum_{q \in Q \backslash\{\bar{q}\}} \alpha_{q}-(r+1)-\gamma \leq 0, \forall \bar{q} \in Q, 0 \leq \alpha_{q} \leq 1, \forall q \in Q\right\} .
\end{aligned}
$$

The following results hold.

(a) If $|Q| \leq 2$, one of the optimal solutions of problem (12) is $(\boldsymbol{e},-r)$;

(b) If $|Q| \geq 3$, one of the optimal solutions of problem (12) is

$$
\begin{cases}\left(e^{d},-r\right), & \text { if } \frac{\sum_{q \in Q} \bar{x}_{q}}{|Q|-1} \leq \bar{x}_{d} ; \\ \left(\frac{1}{|Q|-1} e,-r\right), & \text { if } \bar{x}_{d}<\frac{\sum_{q \in Q} \bar{x}_{q}}{|Q|-1} \leq 1 ; \\ (\boldsymbol{e},-r+|Q|-2), & \text { if } \frac{\sum_{q \in Q} \bar{x}_{q}}{|Q|-1}>1 .\end{cases}
$$

Proof If $|Q| \leq 2$, by removing the redundant constraints that are dominated by $-r-\gamma \leq 0$ and $\alpha_{q} \leq 1, q \in Q$, problem (39) is equivalent to

$$
\max _{\alpha, \gamma}\left\{\sum_{q \in Q} \bar{x}_{q} \alpha_{q}-\bar{y}_{1}-\gamma:-r-\gamma \leq 0,0 \leq \alpha_{q} \leq 1, \forall q \in Q\right\} .
$$

Clearly, $(\boldsymbol{e},-r)$ is an optimal solution. This proves case (a) in the statement.

Next, we consider the case $|Q| \geq 3$. Since the coefficient of $\gamma$ in the objective function in problem (39) is -1 , optimality of problem (39) requires that $\gamma$ must be equal to $-r$, or $\sum_{q \in Q \backslash\left\{q^{\prime}\right\}} \alpha_{q}-(r+1)$ for some $q^{\prime} \in Q$. We have the following two cases.

1) $\gamma=-r$. By eliminating the variable $\gamma$, problem (39) reduces to

$$
\max _{\alpha}\left\{\sum_{q \in Q} \bar{x}_{q} \alpha_{q}-\bar{y}_{1}+r: \sum_{q \in Q \backslash\{\bar{q}\}} \alpha_{q} \leq 1, \forall \bar{q} \in Q, 0 \leq \alpha_{q} \leq 1, \forall q \in Q\right\} .
$$

From the linear programming theory, there are at least $|Q|$ constraints in the above problem being active at the basic optimal solution. 
1.1) If $\sum_{q \in Q \backslash\{\bar{q}\}} \alpha_{q}=1$ for all $\bar{q} \in Q$, we have $\alpha_{q}=\frac{1}{|Q|-1}$ for all $q \in Q$, and $\left(\frac{1}{|Q|-1} e,-r\right)$ is an optimal solution of problem (39).

1.2) Otherwise, we have $\alpha_{q^{\prime}}=0$ or $\alpha_{q^{\prime}}=1$ for some $q^{\prime} \in Q$. In any case, there must exist some $q^{\prime \prime} \in Q$ such that $\alpha_{q^{\prime \prime}}=0$. Together with $\sum_{q \in Q \backslash\left\{q^{\prime \prime}\right\}} \alpha_{q} \leq 1$, it can be easily verified that $\left(e^{d},-r\right)$ is an optimal solution of problem (39).

2) $\gamma=\sum_{q \in Q \backslash\left\{q^{\prime}\right\}} \alpha_{q}-(r+1)$ for some $q^{\prime} \in Q$. By eliminating the variable $\gamma$, problem (39) reduces to

$$
\begin{gathered}
\max _{\alpha}\left\{-\sum_{q \in Q \backslash\left\{q^{\prime}\right\}}\left(1-\bar{x}_{q}\right) \alpha_{q}+\bar{x}_{q^{\prime}} \alpha_{q^{\prime}}-\bar{y}_{1}+(r+1): \sum_{q \in Q \backslash\left\{q^{\prime}\right\}} \alpha_{q} \geq 1,\right. \\
\left.\alpha_{q} \geq \alpha_{q^{\prime}}, \forall q \in Q \backslash\left\{q^{\prime}\right\}, 0 \leq \alpha_{q} \leq 1, \forall q \in Q\right\} .
\end{gathered}
$$

2.1) If $\alpha_{q^{\prime}}=0$, the constraints $\alpha_{q} \geq \alpha_{q^{\prime}}, q \in Q \backslash\left\{q^{\prime}\right\}$, are redundant. This implies that $\left(\boldsymbol{e}^{\boldsymbol{d}^{\prime}},-r\right)$ is an optimal solution of problem (40) where $d^{\prime} \in \operatorname{argmax}_{q \in Q \backslash\left\{q^{\prime}\right\}}\left\{\bar{x}_{q}\right\}$. We note that by the definition of $d$ in (17), the objective value of problem (39) at point $\left(e^{d^{\prime}},-r\right)$ cannot be better than that at point $\left(e^{d},-r\right)$.

2.2) If $\alpha_{q^{\prime}}=1$, we have $\alpha_{q}=1$, for all $q \in Q \backslash\left\{q^{\prime}\right\}$. Then $(\boldsymbol{e},-r+|Q|-2)$ is an optimal solution of problem (40).

2.3) If $0<\alpha_{q^{\prime}}<1$, we have $\alpha_{q} \geq \alpha_{q^{\prime}}>0$ for all $q \in Q \backslash\left\{q^{\prime}\right\}$. There exists a basic optimal solution such that at least $|Q|$ constraints in problem (40) are active. By a simple analysis, the active constraints must be $\sum_{q \in Q \backslash\left\{q^{\prime}\right\}} \alpha_{q}=1$ and $\alpha_{q}=\alpha_{q^{\prime}}$ for all $q \in Q \backslash\left\{q^{\prime}\right\}$. This implies $\alpha_{q}=\frac{1}{|Q|-1}$ for all $q \in Q$, and hence $\left(\frac{1}{|Q|-1} e,-r\right)$ is an optimal solution of problem (39).

In summary, for problem (39), there are three potentially optimal solutions $\left(\boldsymbol{e}^{\boldsymbol{d}},-r\right),\left(\frac{1}{|Q|-1} \boldsymbol{e},-r\right)$, and $(\boldsymbol{e},-r+|Q|-2)$ with the objective value $\bar{x}_{d}-$ $\bar{y}_{1}+r, \frac{1}{|Q|-1} \sum_{q \in Q} \bar{x}_{q}-\bar{y}_{1}+r$, and $\sum_{q \in Q} \bar{x}_{q}-\bar{y}_{1}+r-|Q|+2$, respectively. Finally, comparing these three values, we have case (b) in the statement. This completes the proof.

\section{Proof of Proposition 6}

Proof From the definition of $r$ in (16), we have $(\mathbf{0}, r) \in X$. This, together with assumption (ii) and condition (19), implies that

$$
\begin{aligned}
X=\{(\mathbf{0}, r)\} \cup\left\{(x, k) \in\{0,1\}^{|Q|} \times \mathbb{Z}_{+}: x \neq \boldsymbol{e}, k=r+1\right\} \\
\cup\left\{(x, k) \in\{0,1\}^{|Q|} \times \mathbb{Z}_{+}: k \geq r+2\right\} .
\end{aligned}
$$

The vertices of polyhedron $P$, i.e., $\operatorname{conv}(X)$, are $(\mathbf{0}, r),(x, r+1)$ for all $x \in\{0,1\}^{|Q|}$ with $x \neq \mathbf{0}$ and $x \neq \boldsymbol{e}$, and $(\boldsymbol{e}, r+2)$. Similar to the proof 
in Proposition 5, by removing redundant constraints and using Lemma 2 and Theorem 1, problem (12) reduces to problem (39). Hence, if $|Q| \leq 2$, by Lemma 4, the optimal solution $(\boldsymbol{e},-r)$ of problem (39) corresponds to the inequality $\sum_{q \in Q} x_{q} \leq y_{1}-r$ of polyhedron $P$. The associated face $\{(x, y) \in$ $\left.P: \sum_{q \in Q} x_{q}=y_{1}-r\right\}$ contains $|Q|+1$ affinely independent points: $(\mathbf{0}, r)$ and $\left(e^{q}, r+1\right)$ for each $q \in Q$, which shows that inequality $\sum_{q \in Q} x_{q} \leq y_{1}-r$ defines a facet of polyhedron $P$. This proves case (a) in the statement.

Analogously, if $|Q| \geq 3$, by Lemma 4 , points $\left(\boldsymbol{e}^{d},-r\right),\left(\frac{1}{|Q|-1} \boldsymbol{e},-r\right)$, and $(\boldsymbol{e},-r+|Q|-2)$ are three potentially optimal solutions for problem (39) which correspond to inequalities $x_{d} \leq y_{1}-r, \frac{1}{|Q|-1} \sum_{q \in Q} x_{q} \leq y_{1}-r$, and $\sum_{q \in Q} x_{q} \leq$ $y_{1}-r+|Q|-2$, respectively. To prove that each of the three inequalities defines a facet of polyhedron $P$, we list the $|Q|+1$ affinely independent points in polyhedron $P$ fulfilling them at equality in the following.

\begin{tabular}{|l|l|}
\hline$x_{d} \leq y_{1}-r$ & $(\mathbf{0}, r),\left(e^{d}, r+1\right),\left(e^{d}+e^{q}, r+1\right)$ for each $q \in Q \backslash\{d\}$ \\
\hline$\frac{1}{|Q|-1} \sum_{q \in Q} x_{q} \leq y_{1}-r$ & $(\mathbf{0}, r),\left(\boldsymbol{e}-\boldsymbol{e}^{q}, r+1\right)$ for each $q \in Q$ \\
\hline$\sum_{q \in Q} x_{q} \leq y_{1}-r+|Q|-2$ & $\left(\boldsymbol{e}-\boldsymbol{e}^{q}, r+1\right)$ for each $q \in Q,(e, r+2)$ \\
\hline
\end{tabular}

Thus, we have case (b) in the statement. This completes the proof.

\section{Proof of Proposition 7}

Proof From the definition of $r$ in (16), we have $\left(\mathbf{0}, r \boldsymbol{f}^{\mathbf{1}}\right) \in X$. Combining with assumptions (ii), (iii), and condition (18), we can write set $X$ as:

$$
\begin{aligned}
X=\left\{\left(\mathbf{0}, r \boldsymbol{f}^{\mathbf{1}}\right)\right\} \bigcup\left\{(x, k) \in\{0,1\}^{|Q|} \times \mathbb{Z}_{+}^{|T|}: k_{1} \geq r+1,\right. & \left.\sum_{t \in T \backslash\{1\}} k_{t}=0\right\} \\
& \bigcup\left\{(x, k) \in\{0,1\}^{|Q|} \times \mathbb{Z}_{+}^{|T|}: \sum_{t \in T \backslash\{1\}} k_{t} \geq 1\right\} .
\end{aligned}
$$

Clearly, if $r=0$, the vertices of polyhedron $P$, i.e., conv $(X)$, are $\left(\mathbf{0}, r \boldsymbol{f}^{\mathbf{1}}\right)$, $\left(x,(r+1) \boldsymbol{f}^{\mathbf{1}}\right)$ for all $x \in\{0,1\}^{|Q|}$ with $x \neq \mathbf{0}$, and $\left(x, \boldsymbol{f}^{\boldsymbol{t}}\right)$ for all $x \in\{0,1\}^{|Q|}$ with $x \neq \mathbf{0}$ and $t \in T \backslash\{1\}$. If $r>0$, the additional vertices of polyhedron $P$ are $\left(\mathbf{0}, \boldsymbol{f}^{\boldsymbol{t}}\right)$ for all $t \in T \backslash\{1\}$. Similar to the proof in Proposition 5 , by removing redundant constraints and using Lemma 2 and Theorem 1, problem 
(12) reduces to

$$
\begin{aligned}
& \max _{\alpha, \beta, \gamma}\left\{\sum_{q \in Q} \bar{x}_{q} \alpha_{q}-\bar{y}_{1}-\sum_{t \in T \backslash\{1\}} \bar{y}_{t} \beta_{t}-\gamma:-r-\gamma \leq 0,\right. \\
& \sum_{q \in Q} \alpha_{q}-(r+1)-\gamma \leq 0, \sum_{q \in Q} \alpha_{q}-\beta_{t}-\gamma \leq 0, \forall t \in T \backslash\{1\}, \\
&\left.\beta_{1}=1, \beta_{t} \geq 0, \forall t \in T \backslash\{1\}, 0 \leq \alpha_{q} \leq 1, \forall q \in Q\right\} .
\end{aligned}
$$

We now relax the bound constraints $\beta_{t} \geq 0$ for all $t \in T \backslash\{1\}$ in problem (41). Then as the objective coefficient of $\beta_{t}$ in problem (41) is $-\bar{y}_{t} \leq 0$, we have $\beta_{t}=\sum_{q \in Q} \alpha_{q}-\gamma$ for all $t \in T \backslash\{1\}$ in the relaxation problem. Substituting them into the objective function and dividing the objective function by the positive value $1-\sum_{t \in T \backslash\{1\}} \bar{y}_{t}$, we obtain an equivalent relaxation problem:

$$
\begin{aligned}
& \max _{\alpha, \gamma}\left\{\sum_{q \in Q} \frac{\bar{x}_{q}-\sum_{t \in T \backslash\{1\}} \bar{y}_{t}}{1-\sum_{t \in T \backslash\{1\}} \bar{y}_{t}} \alpha_{q}-\gamma-\frac{\bar{y}_{1}}{1-\sum_{t \in T \backslash\{1\}} \bar{y}_{t}}:\right. \\
& \left.\quad-r-\gamma \leq 0, \quad \sum_{q \in Q} \alpha_{q}-(r+1)-\gamma \leq 0,0 \leq \alpha_{q} \leq 1, \forall q \in Q\right\} .
\end{aligned}
$$

If, for some $q \in Q$, variable $\alpha_{q}$ 's objective coefficient $\frac{\bar{x}_{q}-\sum_{t \in T \backslash\{1\}} \bar{y}_{t}}{1-\sum_{t \in T \backslash\{1\}} \bar{y}_{t}} \leq 0$, then there must exist an optimal solution of problem (42) such that $\alpha_{q}=0$. Hence, we can remove the variables $\alpha_{q}$ with nonpositive objective coefficients ( $q \in Q \backslash \tilde{Q}$ where $\tilde{Q}$ is defined in (20)) from problem (42) and concentrate on the equivalent form of problem (42):

$$
\begin{aligned}
& \max _{\alpha, \gamma}\left\{\sum_{q \in \tilde{Q}} \frac{\bar{x}_{q}-\sum_{t \in T \backslash\{1\}} \bar{y}_{t}}{1-\sum_{t \in T \backslash\{1\}} \bar{y}_{t}} \alpha_{q}-\gamma-\frac{\bar{y}_{1}}{1-\sum_{t \in T \backslash\{1\}} \bar{y}_{t}}:\right. \\
& \left.\quad-r-\gamma \leq 0, \sum_{q \in \tilde{Q}} \alpha_{q}-(r+1)-\gamma \leq 0,0 \leq \alpha_{q} \leq 1, \forall q \in \tilde{Q}\right\} .
\end{aligned}
$$

We have two following cases.

1) $\tilde{Q}=\varnothing$. It can be easily verified that point $(\alpha, \gamma)=(\mathbf{0},-r)$ is optimal for problem (42). Together with $\beta_{t}=\sum_{q \in Q} \alpha_{q}-\gamma=r \geq 0$ for all $t \in$ $T \backslash\{1\}$, we know that $\left(\mathbf{0}, \boldsymbol{f}^{\mathbf{1}}+r \sum_{t \in T \backslash\{1\}} \boldsymbol{f}^{t},-r\right)$ is an optimal solution for problem (41) corresponding to the inequality $0 \leq y_{1}+r \sum_{t \in T \backslash\{1\}} y_{t}-r$ of polyhedron $P$. Moreover, the inequality defines a facet of polyhedron $P$ since the $|Q|+|T|$ affinely independent points $\left(\mathbf{0}, r \boldsymbol{f}^{\mathbf{1}}\right),\left(\mathbf{0}, \boldsymbol{f}^{\boldsymbol{t}}\right)$ for each $t \in T \backslash\{1\}$, and $\left(\boldsymbol{e}^{\boldsymbol{q}}, \boldsymbol{f}^{\boldsymbol{t}^{\prime}}\right)$ for each $q \in Q$ and some $t^{\prime} \in T \backslash\{1\}$, are on the face $\left\{(x, y) \in P: 0=y_{1}+r \sum_{t \in T \backslash\{1\}} y_{t}-r\right\}$. 
2) $\tilde{Q} \neq \varnothing$. Notice that problem (42) are a form of problem (37) and hence by Lemma 3 , point $(\alpha, \gamma)=\left(\boldsymbol{e}^{\boldsymbol{d}},-r\right)$ is optimal for problem (42) where $d$ is defined in (17). Furthermore, for all $t \in T$, we have $\beta_{t}=\sum_{q \in Q} \alpha_{q}-\gamma=$ $r+1 \geq 0$ showing that $\left(\boldsymbol{e}^{\boldsymbol{d}}, \boldsymbol{f}^{\mathbf{1}}+(r+1) \sum_{t \in T \backslash\{1\}} \boldsymbol{f}^{\boldsymbol{t}},-r\right)$ is an optimal solution of problem (41) which corresponds to the inequality $x_{d} \leq y_{1}+$ $(r+1) \sum_{t \in T \backslash\{1\}} y_{t}-r$ for $P$. Finally, the $|Q|+|T|$ affinely independent points $\left(\mathbf{0}, r \boldsymbol{f}^{\mathbf{1}}\right),\left(\boldsymbol{e}^{\boldsymbol{d}},(r+1) \boldsymbol{f}^{\mathbf{1}}\right),\left(\boldsymbol{e}^{\boldsymbol{d}}+\boldsymbol{e}^{\boldsymbol{q}},(r+1) \boldsymbol{f}^{\mathbf{1}}\right)$ for each $q \in Q \backslash\{d\}$, and $\left(\boldsymbol{e}^{\boldsymbol{d}}, \boldsymbol{f}^{\boldsymbol{t}}\right)$ for each $t \in T \backslash\{1\}$ are on the face $\left\{(x, y) \in P: x_{d}=y_{1}+\right.$ $\left.(r+1) \sum_{t \in T \backslash\{1\}} y_{t}-r\right\}$, which implies that the inequality $x_{d} \leq y_{1}+(r+$ 1) $\sum_{t \in T \backslash\{1\}} y_{t}-r$ is facet-defining for polyhedron $P$.

\section{Proof of Proposition 8}

Proof From the definition of $r$ in (16), we have $\left(\mathbf{0}, r \boldsymbol{f}^{\mathbf{1}}\right) \in X$. It follows from assumptions (ii), (iii) and condition (19) that the set $X$ can be equivalently written as:

$$
\begin{aligned}
& X=\left\{\left(\mathbf{0}, r \boldsymbol{f}^{\mathbf{1}}\right)\right\} \bigcup\{\left.(x, k) \in\{0,1\}^{|Q|} \times \mathbb{Z}_{+}^{|T|}: x \neq \boldsymbol{e}, k_{1}=r+1, \sum_{t \in T \backslash\{1\}} k_{t}=0\right\} \\
& \bigcup\left\{(x, k) \in\{0,1\}^{|Q|} \times \mathbb{Z}_{+}^{|T|}: k_{1} \geq r+2, \sum_{t \in T \backslash\{1\}} k_{t}=0\right\} \\
& \bigcup\left\{(x, k) \in\{0,1\}^{|Q|} \times \mathbb{Z}_{+}^{|T|}: \sum_{t \in T \backslash\{1\}} k_{t} \geq 1\right\} .
\end{aligned}
$$

Clear, if $r=0$, the vertices of polyhedron $P$, i.e., conv $(X)$, are $\left(\mathbf{0}, r \boldsymbol{f}^{\mathbf{1}}\right)$, $\left(x,(r+1) \boldsymbol{f}^{\mathbf{1}}\right)$ for all $x \in\{0,1\}^{|Q|}$ with $x \neq \mathbf{0}$ and $x \neq \boldsymbol{e},\left(\boldsymbol{e},(r+2) \boldsymbol{f}^{\mathbf{1}}\right)$, and $\left(x, \boldsymbol{f}^{t}\right)$ for all $x \in\{0,1\}^{|Q|}$ with $x \neq \mathbf{0}$ and for all $t \in T \backslash\{1\}$. If $r>0$, the additional vertices of polyhedron $P$ are $\left(\mathbf{0}, \boldsymbol{f}^{\boldsymbol{t}}\right)$ for all $t \in T \backslash\{1\}$. Similar to the proof in Proposition 5, by removing redundant constraints and using Lemma 2 and Theorem 1, problem (12) reduces to

$$
\begin{aligned}
& \max _{\alpha, \beta, \gamma}\left\{\sum_{q \in Q} \bar{x}_{q} \alpha_{q}-\bar{y}_{1}-\sum_{t \in T \backslash\{1\}} \bar{y}_{t} \beta_{t}-\gamma:-r-\gamma \leq 0,\right. \\
& \sum_{q \in Q \backslash\{\bar{q}\}} \alpha_{q}-(r+1)-\gamma \leq 0, \forall \bar{q} \in Q, \quad \sum_{q \in Q} \alpha_{q}-\beta_{t}-\gamma \leq 0, \forall t \in T \backslash\{1\}, \\
& \left.\beta_{1}=1, \beta_{t} \geq 0, \forall t \in T \backslash\{1\}, 0 \leq \alpha_{q} \leq 1, \forall q \in Q\right\} .
\end{aligned}
$$

We now consider the relaxation of problem (44) obtained by relaxing the bound constraints $\beta_{t} \geq 0$ for all $t \in T \backslash\{1\}$. As the objective coefficient of $\beta_{t}$ in problem (44) is $-\bar{y}_{t} \leq 0$, we can set $\beta_{t}=\sum_{q \in Q} \alpha_{q}-\gamma$ for all $t \in T \backslash\{1\}$ in 
the relaxation problem. In analogy to the proof in Proposition 7, substituting them into the objective function and dividing the objective function by the positive value $1-\sum_{t \in T \backslash\{1\}} \bar{y}_{t}$, this relaxation problem reduces to:

$$
\begin{aligned}
& \max _{\alpha, \gamma}\left\{\sum_{q \in Q} \frac{\bar{x}_{q}-\sum_{t \in T \backslash\{1\}} \bar{y}_{t}}{1-\sum_{t \in T \backslash\{1\}} \bar{y}_{t}} \alpha_{q}-\gamma-\frac{\bar{y}_{1}}{1-\sum_{t \in T \backslash\{1\}} \bar{y}_{t}}:-r-\gamma \leq 0,\right. \\
&\left.\sum_{q \in Q \backslash\{\bar{q}\}} \alpha_{q}-(r+1)-\gamma \leq 0, \forall \bar{q} \in Q, 0 \leq \alpha_{q} \leq 1, \forall q \in Q\right\} .
\end{aligned}
$$

1) $\tilde{Q} \neq Q$. For $q \in Q \backslash \tilde{Q}$ where $\tilde{Q}$ is defined in (20), since $\frac{\bar{x}_{q}-\sum_{t \in T \backslash\{1\}} \bar{y}_{t}}{1-\sum_{t \in T \backslash\{1\}} \bar{y}_{t}}<0$, we can set $\alpha_{q}=0$ in problem (45). This, together with $\alpha_{q} \geq 0$ for all $q \in \tilde{Q}$, implies that the $|Q|$ constraints $\sum_{q \in Q \backslash\{\bar{q}\}} \alpha_{q}-(r+1)-\gamma \leq 0$, for all $\bar{q} \in Q$, in problem (45), can be reduced to a single constraint $\sum_{q \in \tilde{Q}} \alpha_{q}-$ $(r+1)-\gamma \leq 0$ since they are either equivalent to or dominated by this constraint. Therefore, in this case, problem (45) reduces to problem (43), and repeating 1) and 2) in the proof of Proposition 7, we have cases (a) and (b) in the statement.

2) $\tilde{Q}=Q$ and $|Q| \leq 2$. By Lemma 4 , point $(\boldsymbol{e},-r)$ is optimal for problem (45). For each $t \in T \backslash\{1\}$, we have $\beta_{t}=\sum_{q \in Q} \alpha_{q}-\gamma=r+|Q| \geq 0$ showing that $\left(\boldsymbol{e}, \boldsymbol{f}^{\mathbf{1}}+(r+|Q|) \sum_{t \in T \backslash\{1\}} \boldsymbol{f}^{t},-r\right)$ is an optimal solution of problem (44) corresponding to the inequality $\sum_{q \in Q} x_{q} \leq y_{1}+(r+|Q|) \sum_{t \in T \backslash\{1\}} y_{t}-r$ for polyhedron $P$. Moreover, to show that it is facet-defining for polyhedron $P$, we list the $|Q|+|T|$ affinely independent points in polyhedron $P$ satisfying it on equality: $\left(\mathbf{0}, r \boldsymbol{f}^{\mathbf{1}}\right),\left(\boldsymbol{e}^{\boldsymbol{q}},(r+1) \boldsymbol{f}^{\mathbf{1}}\right)$ for each $q \in Q$, and $\left(\boldsymbol{e}, \boldsymbol{f}^{\boldsymbol{t}}\right)$ for each $t \in T \backslash\{1\}$. Thus, we have case (c) in the statement.

3) $\tilde{Q}=Q$ and $|Q| \geq 3$. By Lemma 4 , one of the three points $\left(\boldsymbol{e}^{d},-r\right)$, $\left(\frac{1}{|Q|-1} e,-r\right)$, and $(e,-r+|Q|-2)$ are optimal for problem (45). Using these three points to compute $\beta_{t}=\sum_{q \in Q} \alpha_{q}-\gamma$, for all $t \in T \backslash\{1\}$, we have $\beta_{t}=r+1, r+\frac{|Q|}{|Q|-1}$, and $r+2$, respectively. In all three cases, we have $\beta_{t} \geq 0$ for all $t \in T \backslash\{1\}$ and hence one of the three points $\left(e^{d}, f^{1}+\right.$ $\left.(r+1) \sum_{t \in T \backslash\{1\}} f^{t},-r\right),\left(\frac{1}{|Q|-1} e, f^{1}+\left(r+\frac{|Q|}{|Q|-1}\right) \sum_{t \in T \backslash\{1\}} f^{t},-r\right)$, and $\left(\boldsymbol{e}, \boldsymbol{f}^{1}+(r+2) \sum_{t \in T \backslash\{1\}} \boldsymbol{f}^{t},-r+|Q|-2\right)$ must be optimal for problem (44). Finally, the following table shows that the associated inequalities define facets of polyhedron $P$. 


\begin{tabular}{|c|c|}
\hline$x_{d} \leq y_{1}+(r+1) \sum_{y \in T \backslash\{1\}} y_{t}-r$ & $\begin{array}{l}\left(\mathbf{0}, r \boldsymbol{f}^{\mathbf{1}}\right),\left(\boldsymbol{e}^{\boldsymbol{d}},(r+1) \boldsymbol{f}^{\mathbf{1}}\right) \\
\left(\boldsymbol{e}^{\boldsymbol{d}}+\boldsymbol{e}^{\boldsymbol{q}},(r+1) \boldsymbol{f}^{\mathbf{1}}\right) \text { for each } q \in Q \backslash\{d\} \\
\left(\boldsymbol{e}^{\boldsymbol{d}}, \boldsymbol{f}^{\boldsymbol{t}}\right) \text { for each } t \in T \backslash\{1\}\end{array}$ \\
\hline $\begin{array}{l}\frac{1}{|Q|-1} \sum_{q \in Q} x_{q} \leq \\
y_{1}+\left(r+\frac{|Q|}{|Q|-1}\right) \sum_{y \in T \backslash\{1\}} y_{t}-r\end{array}$ & $\begin{array}{l}\left(\mathbf{0}, r \boldsymbol{f}^{\mathbf{1}}\right),\left(\boldsymbol{e}-\boldsymbol{e}^{q},(r+1) \boldsymbol{f}^{\mathbf{1}}\right) \text { for each } q \in Q, \\
\left(\boldsymbol{e}, \boldsymbol{f}^{t}\right) \text { for each } t \in T \backslash\{1\} .\end{array}$ \\
\hline $\begin{array}{l}\sum_{q \in Q} x_{q} \leq y_{1}+ \\
(r+2) \sum_{y \in T \backslash\{1\}} y_{t}-r+|Q|-2\end{array}$ & $\begin{array}{l}\left(\boldsymbol{e}-\boldsymbol{e}^{\boldsymbol{q}},(r+1) \boldsymbol{f}^{\mathbf{1}}\right) \text { for each } q \in Q \\
\left(\boldsymbol{e}, \boldsymbol{f}^{\boldsymbol{t}}\right) \text { for each } t \in T \backslash\{1\},\left(\boldsymbol{e},(r+2) \boldsymbol{f}^{\mathbf{1}}\right)\end{array}$ \\
\hline
\end{tabular}

Thus, we have case (d) in the statement. This completes the proof. 